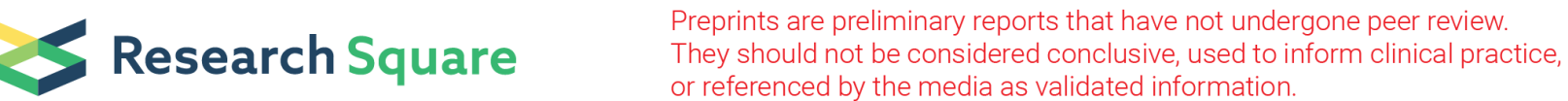

\section{Melatonin Inhibits the Ferroptotic Pathway in Rat Bone Marrow Mesenchymal Stem Cells by Activating the PI3K-AKT-mTOR Signaling Axis to Attenuate Steroid-induced Osteoporosis}

meng li

USTC: University of Science and Technology of China ning yang

USTC: University of Science and Technology of China li hao

USTC: University of Science and Technology of China wei zhou

USTC: University of Science and Technology of China lei li

USTC: University of Science and Technology of China lei liu

USTC: University of Science and Technology of China fang fang

Anhui Medical University

lei $x u$

USTC: University of Science and Technology of China gang yao

USTC: University of Science and Technology of China chen zhu

USTC: University of Science and Technology of China wei Xu

USTC: University of Science and Technology of China shiyuan fang ( $\square$ fangsyustc2@163.com )

USTC: University of Science and Technology of China

\section{Research}

Keywords: Melatonin, Glucocorticoids, Ferroptosis, Steroid-induced osteoporosis

Posted Date: September 2nd, 2021 
DOl: https://doi.org/10.21203/rs.3.rs-850531/v1

License: (c) (1) This work is licensed under a Creative Commons Attribution 4.0 International License. Read Full License 


\section{Abstract}

\section{Objectives}

Steroid-induced osteoporosis (SIOP) is a secondary osteoporosis, which is a systemic bone disease characterized by low bone mass, bone microstructure damage, increased bone fragility, and easy fracture. However, the specific mechanism remains unclear. Glucocorticoid-induced death of osteoblasts and bone marrow mesenchymal stem cells (BMSCs) is an important factor in SIOP. Ferroptosis is an irondependent programmed cell death that differs from apoptosis, cell necrosis, and autophagy, which can be induced by many factors. Herein, we aimed to explore whether glucocorticoids (GCs) cause ferroptosis in BMSCs and determine possible treatment pathways and mechanisms of action. Melatonin (MT), a hormone secreted by the pineal gland, displays strong antioxidant abilities to scavenge free radicals and alleviates ferroptosis in many tissues and organs.

Methods

In this study, we used high-dose dexamethasone (DEX) to observe whether glucocorticoids induced ferroptosis in BMSCs. We then assessed whether MT can inhibit the ferroptotic pathway, thereby providing early protection against GC-induced SIOP, and investigated the signaling pathways involved.

Results

In vitro experiments showed that MT intervention significantly improved GC-induced ferroptosis in BMSCs and significantly improved SIOP in vivo. Pathway analysis showed that MT improves ferroptosis by activating the phosphatidylinositol 3-kinase (PI3K)/protein kinase B (AKT)/mammalian target of rapamycin (mTOR) axis. MT upregulates expression of $\mathrm{PI} 3 \mathrm{~K}$, which is an important regulator of ferroptosis resistance. PI3K activators mimic the anti-ferroptosis effect of MT, but after blocking the PI3K pathway, the effect of MT is weakened. Obviously, MT can protect against SIOP induced by GC. Notably, even after GC-induced ferroptosis begins, MT can confer protection against SIOP.

Conclusion

Our research confirms that GC-induced ferroptosis is closely related to SIOP. Melatonin can inhibit ferroptosis by activating the PI3K-AKT-mTOR signaling pathway, thereby reducing the occurrence of steroid-induced osteoporosis. Therefore, MT may provide a novel strategy for preventing and treating SIOP.

\section{Introduction}

Steroids are a large class of anti-inflammatory agents usually used for inflammation-related syndromes and have effective therapeutic effects[1]. However, the widespread use of these drugs has caused serious side effects. In recent decades, the prevalence of steroid-induced osteoporosis (SIOP) has increased rapidly and has shown a trend toward younger patients[2]. Osteoporosis can lead to a substantial 
increase in the incidence of fragility fractures of the spine and extremities and increase treatment costs for patients[3,4]. However, there is no effective clinical strategy for the prevention and treatment of SIOP[5].

The mechanism of SIOP induced by glucocorticoids (GCs) is unclear. However, a decreased number of osteoblasts is one of the main features of SIOP[6]. Bone marrow mesenchymal stem cells (BMSCs) are the main source of osteoblast differentiation in bones[7]. Studies have shown that the excessive use of steroids can cause oxidative stress in BMSCs. Under severe oxidative stress conditions, reactive oxygen species (ROS) accumulation in cells leads to increased endoplasmic reticulum stress, mitochondrial DNA (mtDNA) damage, mitochondrial dysfunction, and ultimately cell death[8].

Ferroptosis is a recently discovered programmed cell death caused by iron metabolism disorders, and is closely related to various bone diseases $[9,10]$. Unlike autophagy and necrosis, ferroptosis is characterized by the inhibition of GPX4 and system Xc', which leads to the destruction of cysteine metabolism and enhanced lipid peroxidation. Finally, the overactive Fenton reaction produces large amounts of ROS, leading to cell death[11, 12]. According to our previous report (Fang Shiyuan et al), after high-dose glucocorticoid administration, downregulation of GPX4 in osteoblasts and enhanced oxidative stress indicate that glucocorticoids may induce ferroptotic effects and participate in pathogenic processes[13]. Therefore, we speculate that reducing the incidence of ferroptosis in BMSCs may have a positive effect on alleviating SIOP.

Melatonin (MT), synthesized from serotonin in the human pineal gland, performs several important functions, such as anti-oxidation, promotion of bone formation, and anti-inflammatory and anti-tumor effects $[14,15]$. Several studies have shown that melatonin is an effective endogenous antioxidant that indirectly stimulates certain antioxidant enzymes, such as superoxide dismutase (SOD) and glutathione peroxidase (GPX)[16-20]. In recent years, researchers have discovered that melatonin can improve osteoporosis by inhibiting hyperglycemia-induced ferroptosis of osteoblasts[21]. However, whether MT can improve SIOP by reversing the ferroptotic pathway of hormone-induced BMSCs, and the specific mechanism, remains unclear.

In this study, we used high-dose dexamethasone (DEX) to observe whether glucocorticoids induced ferroptosis in BMSCs. We then assessed whether MT can inhibit the ferroptotic pathway, thereby providing early protection against GC-induced SIOP, and investigated the signaling pathways involved. Our experimental results clearly show that MT effectively improves GC-induced SIOP by inhibiting the ferroptotic pathway. It is worth noting that our results also indicate that the protective effect of MT is mediated through the PI3K/AKT/mTOR signaling pathway. In summary, MT may represent a new strategy for the prevention and treatment of GC-induced SIOP.

\section{Materials And Methods}

\section{Animal studies}


All methods were implemented in accordance with the guidelines and regulations of animal ethics standards. Forty 8-week-old male Sprague-Dawley (SD) rats (weight: $300 \pm 50 \mathrm{~g}$ ) were obtained from the Experimental Animal Center of the University of Science and Technology of China, and they were randomly divided into four groups: (1) Control group: saline only; (2) SIOP model group: DEX $20 \mathrm{mg} / \mathrm{kg}$ (i.m.) for eight weeks; (3) MT pre-treatment group: MT $50 \mathrm{mg} / \mathrm{kg}$ (i.p.) (Sigma-Aldrich, USA) on the first day, from the second day, rats received DEX $20 \mathrm{mg} / \mathrm{kg}$ (i.m.) + MT $50 \mathrm{mg} / \mathrm{kg}$ (i.p.) for eight weeks, then MT $50 \mathrm{mg} / \mathrm{kg}$ (i.p.) for four weeks; (4) MT post-treatment group: DEX $20 \mathrm{mg} / \mathrm{kg}$ (i.m.) for eight weeks, then MT $50 \mathrm{mg} / \mathrm{kg}$ (i.p.) for four weeks. Finally, the rats in each group were sacrificed under general anesthesia. Surgical instrumentation was used to remove muscle and soft tissue, and the entire femur was removed for subsequent experiments.

\section{Micro-computed tomography (micro-CT) scans}

To evaluate the changes in bone morphology of rats, we used high-resolution micro-CT (SKYSCAN, Alteslar, SKYSCAN 1176, Belgium), with the following scan parameters: $18 \mu \mathrm{m}, 70 \mathrm{kV}$, and $141 \mathrm{~mA}$, and measured the double lateral femur. Subsequently, a CT analyzer (CTAN, Bruker) was used to analyze the images and perform 3D image reconstruction. The morphometric parameters were used to evaluate bone mineral density $\left(\mathrm{BMD} \mathrm{mg} / \mathrm{cm}^{3}\right)$, bone surface/bone volume (BS/BV, \%), bone volume/total volume (BV/TV, \%), and the number of trabecular bones ( Tb.N, 1/mm), trabecular bone spacing (Tb.Sp, mm) and trabecular bone thickness (Tb.Th, $\mathrm{mm}$ ).

\section{Hematoxylin and eosin (H\&E) staining}

The femoral specimens were fixed in $4 \%$ paraformaldehyde for 24 hours and decalcified with $10 \%$ ethylenediaminetetraacetic acid (EDTA, Sigma-Aldrich, USA) for 4 weeks. Thereafter, the decalcified femur samples were embedded in paraffin and cut into $7 \mu \mathrm{m}$-thick sections, and placed on glass slides. Finally, H\&E staining was performed to stain the femoral sections. Briefly, the sections were dehydrated with ethanol, deparaffinized with xylene, stained with hematoxylin for $10 \mathrm{~min}$, and then stained with eosin for $20 \mathrm{~s}$. After rinsing, the samples were sealed with a neutral resin. The staining results were observed under a laser scanning microscope (LSM, Carl Zeiss, Oberkochen, Germany).

\section{Histological and immunohistochemical analysis}

The process of fixation, decalcification, embedding, and slicing of rat femur specimens was the same as that described by H\&E staining. For immunohistochemical staining, the sections were deparaffinized, antigen repaired, and blocked with horse serum for $30 \mathrm{~min}$. Then, in order to identify the differences in the expression of ferroptosis markers in the above four groups of specimens, the following primary antibodies (system xc', ACSL4, FSP1, GPX4) were incubated with samples for 12 hours, followed by incubation with the relevant biotinylated secondary antibody. All antibodies were purchased from (Abcam Cambridge, UK). The staining results were observed under a laser scanning microscope. 


\section{Cell culture}

BMSCs were obtained from the femur and tibia of 6-week-old healthy Sprague-Dawley rats[22]. Freshly extracted BMSCs were cultured in a essential medium (a-MEM, HyClone, Logan, UT, USA), which contains $10 \%$ fetal bovine serum (FBS) and 1\% penicillin/streptomycin. The petri dish containing BMSCs was placed in an incubator at $37{ }^{\circ} \mathrm{C}$ supplied with $5 \% \mathrm{CO}_{2}$. The minimal medium was changed every three days, and BMSCs were sub-cultured. Cells from 3-6 passages were used in this study.

\section{Cell proliferation and toxicity assay}

A cell counting kit (CCK-8) (Beyotime, China) was used to determine the cytotoxicity of DEX and MT. Briefly, BMSCs were cultured at a density of $5 \times 10^{3}$ cells/well in a 96 -well plate. The cells were then incubated with various concentrations of DEX $\left(10^{-5}, 10^{-4}, 10^{-3}\right.$, and $\left.10^{-2} \mathrm{M}\right)$ or MT $(0,5,10,50,100$, and $200 \mu \mathrm{M})$ for 24,48 , and $72 \mathrm{~h}$. To detect BMSC activity, we added a solution containing $90 \%$ serum-free aMEM and $10 \%$ CCK- 8 to each well, and then cultured the cells for $2 \mathrm{~h}$ at $37^{\circ} \mathrm{C}$ in the dark. The absorbance of each well was measured at $450 \mathrm{~nm}$ using a microplate reader (BioTek, Vermont, USA).

\section{Cell death assay}

BMSCs were cultured in three replicate wells of a 24-well plate (cell density: $1 \times 10^{4}$ cells per well) for $72 \mathrm{~h}$. Annexin V-mCherry/SYTOX Green detection kit (C070M, Beyotime, China) was used to detect cell death. The BMSCs were incubated in each well with DEX $\left(10^{-4}, 10^{-3}\right.$, and $\left.10^{-2} \mathrm{M}\right)$ and supplemented with different doses of MT $(10 \mu \mathrm{M}$ and $100 \mu \mathrm{M})$ for $24 \mathrm{~h}$. Cells were incubated in each well with a medium containing $194 \mu \mathrm{L}$ Annexin V-mCherry Binding Buffer, $5 \mu \mathrm{L}$ Annexin V-mCherry, and $1 \mu \mathrm{L}$ SYTOX Green for $20 \mathrm{~min}$ at $37^{\circ} \mathrm{C}$ in the dark. A laser scanning microscope was used to image the results to monitor the level of cell death.

\section{ROS assay}

The cells were cultured in 24-well plates (cell density: $1 \times 10^{4}$ cells/well) for $72 \mathrm{~h}$ in three replicate wells. To detect the amount of intracellular ROS, BMSCs in each well were incubated with a-MEM medium containing a gradient of DEX $\left(10^{-4}, 10^{-3}\right.$, and $\left.10^{-2} \mathrm{M}\right)$ or DEX at a concentration of $10^{-3} \mathrm{M}$ supplemented with different doses of MT (10 and $100 \mu \mathrm{M})$ for $24 \mathrm{~h}$. The cells were incubated with a medium containing 2,7-dichlorofluorescein diacetate (DCFH-DA, 1:1,000 dilution) at $37^{\circ} \mathrm{C}$ for $20 \mathrm{~min}$ in the dark. After subjecting the cells to washing steps three times using a phosphate-buffered solution (PBS), the Olympus IX51 microscope was used to image the results to determine ROS levels. ROS content was determined based on the average fluorescence intensity.

\section{Immunofluorescence assay}

After incubation with the medium without DEX or supplemented with various doses of DEX and MT, the glass slide containing the BMSC sample was rinsed with PBS three times, and fixed in frozen 
paraformaldehyde for 15 min. Next, the sample was incubated with QuickBlockTM IF blocking solution (Beyotime Biotech) for $1 \mathrm{~h}$, and incubated with primary GPX 4 and PI3K antibodies at $4{ }^{\circ} \mathrm{C}$ overnight. After incubation, the sample was rinsed thrice with PBS and incubated with fluorescein isothiocyanate secondary antibody at $37^{\circ} \mathrm{C}$ for $1 \mathrm{~h}$. Finally, 4,6-dimidyl-2-phenylindole (DAPI was used to stain the cell nuclei, and the results were imaged using a laser scanning microscope.

\section{Enzyme-linked immunosorbent assay (ELISA)}

Collect two milliliters of blood from rats in each group and use EDTA for anticoagulation. Serum samples were collected by centrifuging blood samples at $1000 \mathrm{rpm}$ for 20 minutes at $4^{\circ} \mathrm{C}$. The ELISA kit of MT (Abbexa, Cambridge, UK) was used according to the manufacturer's instructions, and the absorbance was measured at $490 \mathrm{~nm}$ or $540 \mathrm{~nm}$ using the ELX800 absorbance microplate reader (Bio-Tec, USA).

\section{Quantitative Reverse Transcription Polymerase Chain Reaction (qRT-PCR)}

After cell intervention, TRIzol reagent (Beyotime, China) was added to extract total RNA from different groups of BMSCs and reverse transcribed into CDNA using a synthesis kit (Takara Bio, Japan). Using a mixture of dNTP reagent (TaKaRa, Japan) and RNase-free $\mathrm{H}_{2} \mathrm{O}$ (Abcam, Cambridge, UK), the gene levels of system xc', GPX4, ACSL4, FSP1, PI3K, AKT, and mTOR were analyzed using qRT-PCR (S1000, Bio-Rad, USA). The $2^{-\triangle \triangle C T}$ method was used to analyze the gene expression data, and the glyceraldehyde phosphate dehydrogenase (GAPDH) gene was used as a housekeeping gene for normalization.

System xc forward: 5'-ATACGCTGAGTGTGGTTTGC-3'

System Xc' reverse: 5'-CTTCATCCACTTCCACAGCG-3'

GPX4 forward: 5'-ATACGCTGAGTGTGGTTTGC-3'

GPX4 reverse: 5'-CTTCATCCACTTCCACAGCG-3'

ACSL4 forward: 5'-TGAATGTCTGCTTCTGCTGC-3'

ACSL 4 reverse: 5'-CCAACTCTTCCAGTAGTGTAGTCGG-3'

FSP1 forward:5'-AGCTTCTTGGGGAAAAGGAC-3'

FSP1 reverse: 5'-CCCCAACCACAT CAGAGG-3'

PI3K forward: 5'-AGTGGGAACCGAAGAAGAAG-3'

PI3K reverse: 5'-GACGGGAACTGACTGGATGAAC-3'

AKT forward: 5'-ATGGACTTCCGGTCAGGTTC-3'

AKT reverse: 5'-GCCCTTGCCCAGTAGCTTCA-3' 
mTOR forward: 5'-GCTTATCAAGCAAGCGACATCTCA-3'

mTOR reverse: 5'-TCCACTGGAAGCACAGACCAAG-3'

GAPDH forward: 5'-TGACCTCAACTACATGGTCTACA-3'

GAPDH reverse: 5'-CTTCCCATTCTCGGCCTTGTACA-3'

\section{Western blotting}

After subsequent processing, BMSCs were harvested and proteins were extracted using radioimmunoprecipitation assay (RIPA) buffer (NCM Biotech, Soochow, China). Proteins were obtained by collecting the supernatant after centrifugation $(14,800 \times g)$ for 25 min at $4{ }^{\circ} \mathrm{C}$. After determination and standardization of the total concentration of protein in each group, samples with equal protein content were separated using $10 \%$ sodium dodecyl sulfate (SDS)-polyacrylamide gel electrophoresis (PAGE) (Beyotime Biotech) and transferred onto nitrocellulose membranes. After blocking nonspecific binding sites using the QuickBlock Blocking Buffer (Beyotime Biotech) for $1 \mathrm{~h}$, the membranes were incubated with primary antibodies overnight at $4{ }^{\circ} \mathrm{C}$. These primary antibodies included System $x C^{-}(1: 5,000)$, ACSL $^{-}$ (1:10,000), FSP1 (1:1,000), GPX4 (1:2,000), PI3K (1:1,000), AKT (1:10,000), and mTOR $(1: 10,000)$. Thereafter, membranes were incubated with the corresponding secondary antibodies $(1: 5,000)$ for $1 \mathrm{~h}$. Finally, we used enhanced chemiluminescence (Pierce ECL) to visualize the protein results, and the relative gray value was measured using Image Lab 3.0.

\section{Statistical analysis}

Statistical software SPSS version 20 was used for analysis, and all results were expressed in the form of mean \pm standard deviation. A one-way analysis of variance (ANOVA) was used to determine the statistical significance. The statistical significance was set as $P<0.05$.

\section{Results}

\section{DEX activates the ferroptotic pathway of BMSCs}

First, the effects of DEX on the proliferation of BMSCs were tested. The results of CCK-8 showed that DEX, at concentrations ranging from $10^{-4} \mathrm{M}$ to $10^{-2} \mathrm{M}$, had a significant inhibitory effect on the proliferation of BMSCs (Figure S1a). To confirm whether DEX affected the ferroptotic pathway of BMSCs, we treated BMSCs with various concentration gradients of DEX and used oxidative stress kits and cell death kits to observe the relevant changes. The results showed that with the increase in DEX concentration, the ROS content in BMSCs increased, especially at concentrations of $10^{-3} \mathrm{M}$ and $10^{-2} \mathrm{M}$ (Figure 1h-i). At the same time, the number of nuclear dead cells in BMSCs also increased significantly (Figure 1j-k). Furthermore, western blotting and qRT-PCR were used to detect the expression of the ferroptosis-related indicators system Xc', ACSL4, GPX4, and FSP1. The results showed that as the 
concentration gradient increased, DEX regulated the levels of system XC', ACSL4, GPX4, and FSP1 to varying degrees (Figure 1a-e). Cell immunofluorescence staining also confirmed the expression of GPX4 in the cytoplasm of BMSCs. We found that the fluorescence intensity of GPX4 in BMSCs after DEX intervention was significantly downregulated (Figure 1f-g). Furthermore, the effects of varying durations of DEX stimulation on the ferroptotic pathway in BMSCs were further examined. Results indicated that ferroptosis induced by DEX was positively correlated with induction time (Figure S2a-i). In order to explore whether MT is associated with SIOP, an ELISA kit was used to detect the change in MT content in the blood of rats after DEX intervention, and the expression of MT receptors in BMSCs was determined. The results showed that the expression of MT decreased with increasing DEX concentration (Figure $2 \mathrm{~b}$ ). In order to verify these in vitro results, we obtained the femoral tissues of the SIOP model group, after rats received DEX (20 mg/kg) treatment for eight weeks, and the control group for comparative study (Figure 2a). Micro-CT images and H\&E staining results showed that, compared with the control group, the trabecula of the model group was sparse and thinner, with wider gaps, and the arrangement was disordered (Figure 2c-d). System xc', GPX4, and FSP1 were detected using immunohistochemical staining. We found that in the SIOP model group, the number of related protein-positive cells decreased significantly (Figure 2e-i). qRT-PCR and western blotting also confirmed that expression levels of ferroptotic pathway-related indicators, system Xc', GPX4, and FSP1, and MT1 in bone tissue were downregulated to varying degrees, while ACSL4 was upregulated (Figure 2j-0). Therefore, these data indicate that DEX induces ferroptosis in the rat SIOP model.

\section{Ferroptosis inhibitor regulates the expression of related proteins in BMSCs}

To further verify whether ferroptosis is one of the main factors of DEX-induced BMSC death, we exposed cells to DEX combined with a ferroptosis inhibitor (Ferrastatin-1, Sigma-Aldrich, USA) and determined ROS levels and cell death. The results showed that with the use of ferroptosis inhibitors, both the ROS content and the number of dead cells showed a significant downward trend (Figure 3f-i). Western blotting and qRT-PCR were used to detect the expression of ferroptosis-related indicators system Xc', ACSL4, GPX4, and FSP1. We found that DEX regulates the levels of system Xc', ACSL4, GPX4, and FSP1 to varying degrees, but the use of ferrastatin-1 can reverse this change to a certain extent (Figure 3a-e). Therefore, these results indicate that DEX-induced ferroptosis is the main cause of BMSC death.

\section{MT can reduce the ferroptosis level in BMSCs induced by DEX}

We investigated whether MT could inhibit the ferroptosis pathway of BMSCs. The CCK-8 results showed that MT concentrations in the range of $5 \mu \mathrm{M}$ to $200 \mu \mathrm{M}$ had no obvious toxic effects on BMSCs (Figure S1b). DEX, at a concentration of $10^{-3} \mathrm{M}$, and DEX supplemented with various MT concentrations $(10,50$, and $100 \mu \mathrm{M}$ ) were incubated with BMSCs for $24 \mathrm{~h}$. The results of ROS staining showed that low-dose (10 $\mu \mathrm{M})$, medium-dose $(50 \mu \mathrm{M})$, and high-dose $(100 \mu \mathrm{M}) \mathrm{MT}$ significantly reduced Ros levels in BMSCs, especially at high doses of MT (Figure $\mathbf{4 f - g}$ ). In addition, using the cell death staining kit revealed that high doses of MT significantly reduced the number of dead cells (Figure 4h-i). Western blotting and qRTPCR further confirmed that MT regulated the levels of ferroptotic pathway-related indicators in BMSCs, 
increased the expression of system Xc', GPX4, and FSP1, and downregulated the expression of ACSL4 (Figure 4a-e). These results indicate that MT inhibited DEX-induced ferroptosis in BMSCs.

\section{MT reduces ferroptosis in DEX-induced BMSCs by activating the PI3K-AKT-mTOR pathway}

The PI3K-AKT pathway is closely related to a variety of orthopedic diseases. It is an intracellular signal transduction pathway, and it also responds to extracellular signals to promote cell proliferation, metabolism and growth. The key genes involved in this process are phosphatidylinositol 3-kinase (PI3K) and protein kinase $B$ (AKT), which are mediated by phosphorylation of serine or threonine on a series of downstream substrates[23-26]. mTOR is a key kinase downstream of PI3K/AKT and is an atypical serine/threonine protein kinase that can integrate various extracellular signals, such as nutrition, energy, and growth factors, and participates in important links such as protein translation, gene transcription and ribosome synthesis. At the same time, mTOR plays a very important role in cell growth, cell apoptosis and special non-programmed death[27-30]. To further understand the ferroptosis mechanism of MT and verify whether MT regulates the effect of DEX on BMSCs by activating the PI3K-AKT-mTOR signaling axis, we used exposed BMSCs to MT at different concentration gradients to observe changes in the expression of factors related to the PI3K-AKT-mTOR pathway. As shown in Figure 5a-d, the results of western blot and qRT-PCR showed that DEX significantly downregulated protein expression of PI3K, AKT, and mTOR. However, after MT supplementation, as the concentration increased, the expression of PI3K, AKT, and mTOR appeared upregulated. Furthermore, immunofluorescence assay results showed that the addition of DEX significantly reduced the fluorescence intensity of PI3K. After MT supplementation, the fluorescence intensity of PI3K in BMSCs was significantly enhanced (Figure $5 \mathrm{e}-\mathrm{f}$ ). These results further indicate that MT activates the PI3K-AKT-mTOR pathway. We then investigated whether intervention in the PI3K pathway could regulate GC-induced ferroptosis. The effect of adding a PI3K agonist (740 Y-P, $10 \mu \mathrm{M}$, MedChemExpress, USA) to BMSCs was observed. As shown in Figure S3a-j, with the activation of PI3K, which mimics the positive regulation of MT, ROS levels were significantly reduced in BMSCs, and the ferroptosis inhibitory effect was significant. To further verify our hypothesis, GDC0941 (a selective inhibitor of PI3K, LC Laboratories,USA) was used to inhibit the expression of PI3K. As shown in Figure 6a$f$, the expression of PI3K, AKT, and mTOR was downregulated when GDC0941 was added. Western blotting and qRT-PCR showed that GDC0941 inhibited the expression of the ferroptosis pathway. The results of ROS staining and the cell death kit showed that with the use of PI3K inhibitors, the positive regulatory effect of MT was significantly weakened, and the number of dead cells increased significantly

(Figure $6 \mathrm{~g}-\mathrm{j}$ ). These analyses indicate that MT can reduce the levels of ferroptosis and oxidative stress in DEX-induced BMSCs by activating the PI3K-AKT-mTOR pathway.

\section{MT attenuated ferroptosis and osteoporosis in the rat SIOP model}

In in vivo experiments, we further verified whether MT affects bone morphology in the early stages of SIOP. Figure 7a illustrates the timeline of the DEX-induced SIOP model and administration of MT in vivo. The results of micro-CT images and H\&E staining showed that bone density was partially restored, the bone trabecula was thicker, and the arrangement was more regular in the pre-treatment group (Figure $7 \mathbf{b}$, 
j). The CT analysis results further confirmed that MT pre-treatment not only increased the BMD, BV/TV, Tb.N, and Tb.Th values, but also significantly reduced the BS/BV and Tb.Sp values compared with that in the model group (Figure 7c-h). System xc-', ACSL4, GPX4, and FSP1 were detected using immunohistochemical and immunofluorescence staining. The results showed that the number of system $\mathrm{XC}^{-}$and FSP1 positive cells in the model group significantly decreased, ACSL4 positive cells increased, and fluorescence intensity of GPX4 decreased compared with the control group, but the MT pre-treatment group displayed significant reversal of these changes (Figure 7i-o). In addition, western blot and qRT-PCR were used to detect the expression of the ferroptotic pathway-related indicators system Xc', ACSL4, GPX4, and FSP1 in the three groups of bone tissues. We found that the expression levels of system Xc, GPX4, and FSP1 in the model group were downregulated to varying degrees, and ACSL4 was upregulated, but pre-treatment with MT significantly improved this change (Figure 7p-t). These results indicate that MT significantly improves ferroptosis and osteoporosis levels in the SIOP model.

\section{Even after DEX-induced ferroptotic effects begin, MT can still improve SIOP}

Finally, we tested whether MT exerted a therapeutic effect on DEX-induced SIOP. Figure 8a shows the process of MT post-treatment in vivo. We found that although the time of the first administration of MT was significantly delayed, bone density was still partially restored (Figure $\mathbf{8 b}-\mathbf{h}, \mathbf{j}$ ). The results of immunohistochemical staining, western blotting, and qRT-PCR further confirmed that even after the bone was exposed to high doses of DEX, MT use still protected BMSCs from ferroptosis (Figure 8i-t). Overall, our results show that MT not only contributes to the prevention of SIOP, but also plays a vital role in treatment.

\section{Discussion}

Melatonin is an effective endogenous antioxidant[31]. The latest report pointed out that melatonin has a protective effect on ferroptosis induced by traumatic brain injury, but this effect is offset by the loss of ferritin $\mathrm{H}$ in neurons[32]. Simultaneously, the study showed that melatonin can ameliorate hypoxicischemic brain injury by activating the Akt/Nrf2/Gpx4 signaling pathway[33]. Some scholars have also studied the relationship between melatonin and ferroptosis in a diabetic osteoporosis model and found that melatonin can inhibit high glucose-induced ferroptosis by activating the Nrf2/HO-1 signaling pathway to treat type 2 diabetic osteoporosis[21]. These reports show that there is a correlation between MT and ferroptosis.

The pathogenesis of SIOP is complicated[34-36]. BMSCs play a key role in normal bone metabolism and are considered ideal seed cells with the potential to maintain and repair bone tissue[37]. Glucocorticoids (GCs) inhibit the growth of BMSCs and are an important way for GC to induce osteoporosis[38, 39]. Our previous studies (Yang Ning, Li Meng, etc.) have found that high concentrations of GC can induce oxidative stress and BMSC death[40]. Several studies have also indicated that oxidative stress is a contributing factor to the pathological process of SIOP, and TXNIP protein can reduce oxidative stress and reverse the process of bone loss by upregulating the MOP pathway[41]. Ferroptosis is caused by the 
inhibition of GPX4 and system xc', which leads to cystine metabolism inhibition, enhanced lipid peroxidation, and iron metabolism dysfunction, and generates a large amount of ROS and triggers cell death[11]. In the present study, we confirmed that DEX activates the ferroptotic pathway, leading to the downregulation of related markers, such as GPX4, system xc', and FSP1 in bone marrow mesenchymal stem cells. Conversely, the observed increase in ROS may be due to increased lipid peroxidation levels induced by DEX, which causes metabolic disorders involving glutamate and cysteine. These results illustrate that DEX induces ferroptotic changes in bone marrow mesenchymal stem cells.

Many studies have indicated that melatonin is closely related to ferroptosis[21, 32, 33, 42]. Considering that MT inhibits ferroptosis in a variety of tissues and organs, it is speculated that melatonin may have a beneficial effect on our SIOP model. In vitro studies have shown that MT can reduce GC-induced ROS and ferroptosis levels. Through micro-CT and H\&E staining analysis, we found that melatonin increased the number of cancellous bone and trabecular bone to a certain extent, increased bone density, narrowed the trabecular space, and the arrangement was more regular. Immunohistological staining results showed that the use of melatonin reduced the number of positive cells for ferroptosis marker proteins such as GPX4, system Xc', and FSP1 in femoral tissue. At the same time, we found that even if SIOP had been formed, the use of MT during the treatment phase produced a certain inhibitory effect on ferroptosis and applied a therapeutic effect on osteoporosis.

The PI3K-AKT-mTOR signaling axis plays an important role in several physiological and pathological processes, including cell proliferation, differentiation, and functional expression[43, 44]. It has been reported that the activation of PI3K can functionally confer ferroptosis inhibitory effects on cancer cells. In terms of mechanism, the generation of this resistance requires continuous activation of mTORC1 and induction of sterol regulatory element binding protein 1 (SREBP1). SREBP1 is an mTORC1-dependent protein, and at the same time acts as an important central transcription factor to regulate lipid metabolism. Monounsaturated fatty acids can mediate the ferroptosis inhibitory activity of SREBP1, and this process is mainly regulated by Stearoyl-CoA desaturase-1 (SCD1), the transcription target of SREBP1.When mTORC1 was inhibited, ferroptosis was suppressed in the PI3K-activated breast cancer xenograft mouse model, and the tumors were almost completely resolved[45]. In our study, we also found that with the intervention of GC on BMSCs, the expression of PI3K and its downstream proteins AKT and mTOR were significantly downregulated, while PI3K agonists inhibited GC-induced ferroptosis.

Recently, it has been reported that MT can alleviate glucocorticoid-induced osteoblast suppression by activating the PI3K/AKT signaling pathway[46]. Our research also showed that the PI3K pathway was activated with the use of MT. Related studies have shown that human melatonin MT1 and MT2 receptors transmit signals through ERK $1 / 2$, and the activation of ERK $1 / 2$ by these receptors is completely dependent on the $\mathrm{G}$ protein. The activation of the signal axis downstream of the $\mathrm{G}$ protein is the same for the two receptors and involves the activation of the PI3K/PKCZ/C-Raf/MEK/ERK signaling axis[47]. Activation of the extracellular regulated protein kinase (ERK) pathway is considered to be one of the signs of ferroptosis, and the amount of ERK-phosphate is positively correlated with the progress of 
ferroptosis[48]. In order to be more precise about the pathway of action of MT, the PI3K inhibitor GDC0941 was used to intervene in GC-induced BMSCs. We found that GDC0941 reversed the effect of MT, further confirming that MT functions by activating the PI3K pathway. However, further research is needed to determine whether ERK1/2 phosphorylation plays a role in the MT-mediated regulation of PI3K expression. Finally, in addition to the PI3K/AKT/mTOR signaling pathway, other signaling pathways and autophagy related to GC-induced SIOP cannot be ignored and should be explored in the future.

\section{Conclusion}

In conclusion, our research confirms that GC-induced ferroptosis is closely related to SIOP. Melatonin can inhibit ferroptosis by activating the PI3K-AKT-mTOR signaling pathway, thereby reducing the occurrence of steroid-induced osteoporosis. Our results indicate that melatonin may have important implications for the prevention and treatment of SIOP. However, further studies are required to verify our findings.

\section{Abbreviations}

SIOP: Steroid-induced osteoporosis;

BMSCs: Bone marrow mesenchymal stem cells;

GCs: Glucocorticoids;

MT: Melatonin; DEX: Dexamethasone;

PI3K: Phosphatidylinositol 3-kinase;

AKT: Protein kinase $B$;

mTOR: Mammalian target of rapamycin;

ROS: Reactive oxygen species;

mtDNA: Mitochondrial DNA;

SOD: Superoxide dismutase;

GPX: Glutathione peroxidase;

SD: Sprague-Dawley;

EDTA: Ethylenediaminetetraacetic acid;

FBS: Fetal bovine serum;

GAPDH: Glyceraldehyde phosphate dehydrogenase; 
RIPA: Radioimmunoprecipitation assay;

SREBP1: Sterol regulatory element binding protein 1;

SCD1: Stearoyl-CoA desaturase-1.

\section{Declarations}

\section{Author contributions}

LM, YN and HL contributed to the conception of the work; LL, LMand FF contributed to the experiments; ZW, XW and ZC contributed to the data acquisition; LM and YG wrote the manuscript; HL, XL and FSY revised the manuscript. All authors contributed to the final approval of the manuscript.

\section{Funding}

This study was supported by the Fundamental Research Funds for the Central Universities WK9110000190 (Meng Li), WK9110000152(Shiyuan Fang), WK9110000178(Lei Li); Natural Science Foundation of Anhui Province 2108085QH317 (Meng Li); National Natural Science Foundation of China (81373421, 81270650) (Chen Zhu).

\section{Availability of data and materials}

All data generated or analyzed during this study are included in this published article. The datasets analyzed during the study are available from the corresponding author on reasonable request.

\section{Ethics approval and consent to participate}

This study was approved by the Medical Ethics Committee of the First Affiliated Hospital of the University of Science and Technology of China, and all methods were implemented in accordance with the guidelines and regulations of animal ethics standards.

\section{Consent for publication}

Not applicable

\section{Competing interests}

The authors declare that they have no competing interests.

\section{References}

[1] V.S. Pelly, A. Moeini, L.M. Roelofsen, E. Bonavita, C.R. Bell, C. Hutton, A. Blanco-Gomez, A. Banyard, C.P. Bromley, E. Flanagan, S.-C. Chiang, C. Jorgensen, T.N. Schumacher, D.S. Thommen, S. Zelenay, Anti- 
inflammatory drugs remodel the tumor immune environment to enhance immune checkpoint blockade efficacy, Cancer Discov, (2021).

[2] D. Grennan, S. Wang, Steroid Side Effects, JAMA, 322 (2019) 282.

[3] P. Chotiyarnwong, E.V. McCloskey, Pathogenesis of glucocorticoid-induced osteoporosis and options for treatment, Nat Rev Endocrinol, 16 (2020) 437-447.

[4] J.E. Compston, M.R. McClung, W.D. Leslie, Osteoporosis, Lancet, 393 (2019) 364-376.

[5] L. Song, L. Cao, R. Liu, H. Ma, Y. Li, Q. Shang, Z. Zheng, L. Zhang, W. Zhang, Y. Han, X. Zhang, H. Yang, Y. Wang, G. Melino, C. Shao, Y. Shi, The critical role of T cells in glucocorticoid-induced osteoporosis, Cell Death Dis, 12 (2020) 45.

[6] L. Buckley, M.B. Humphrey, Glucocorticoid-Induced Osteoporosis, N Engl J Med, 379 (2018) 25472556.

[7] J.P. Bidwell, M.B. Alvarez, M. Hood, P. Childress, Functional impairment of bone formation in the pathogenesis of osteoporosis: the bone marrow regenerative competence, Curr Osteoporos Rep, 11 (2013) 117-125.

[8] F. Zhang, W. Peng, J. Zhang, W. Dong, J. Wu, T. Wang, Z. Xie, P53 and Parkin co-regulate mitophagy in bone marrow mesenchymal stem cells to promote the repair of early steroid-induced osteonecrosis of the femoral head, Cell Death Dis, 11 (2020) 42.

[9] S. Doll, F.P. Freitas, R. Shah, M. Aldrovandi, M.C. da Silva, I. Ingold, A. Goya Grocin, T.N. Xavier da Silva, E. Panzilius, C.H. Scheel, A. Mourão, K. Buday, M. Sato, J. Wanninger, T. Vignane, V. Mohana, M. Rehberg, A. Flatley, A. Schepers, A. Kurz, D. White, M. Sauer, M. Sattler, E.W. Tate, W. Schmitz, A. Schulze, V. O'Donnell, B. Proneth, G.M. Popowicz, D.A. Pratt, J.P.F. Angeli, M. Conrad, FSP1 is a glutathioneindependent ferroptosis suppressor, Nature, 575 (2019) 693-698.

[10] C. Mao, X. Liu, Y. Zhang, G. Lei, Y. Yan, H. Lee, P. Koppula, S. Wu, L. Zhuang, B. Fang, M.V. Poyurovsky, $\mathrm{K}$. Olszewski, B. Gan, DHODH-mediated ferroptosis defence is a targetable vulnerability in cancer, Nature, 593 (2021) 586-590.

[11] J. Zheng, M. Conrad, The Metabolic Underpinnings of Ferroptosis, Cell Metab, 32 (2020) 920-937.

[12] X. Jiang, B.R. Stockwell, M. Conrad, Ferroptosis: mechanisms, biology and role in disease, Nat Rev Mol Cell Biol, 22 (2021) 266-282.

[13] J. Lu, J. Yang, Y. Zheng, X. Chen, S. Fang, Extracellular vesicles from endothelial progenitor cells prevent steroid-induced osteoporosis by suppressing the ferroptotic pathway in mouse osteoblasts based on bioinformatics evidence, Sci Rep, 9 (2019) 16130. 
[14] A. Slomski, Melatonin Improves Sleep in Patients With Circadian Disruption, JAMA, 320 (2018) 749.

[15] A. Karamitri, R. Jockers, Melatonin in type 2 diabetes mellitus and obesity, Nat Rev Endocrinol, 15 (2019) 105-125.

[16] S. Tousi, P. Zoufan, A.R. Ghahfarrokhie, Alleviation of cadmium-induced phytotoxicity and growth improvement by exogenous melatonin pretreatment in mallow (Malva parviflora) plants, Ecotoxicol Environ Saf, 206 (2020) 111403.

[17] E. Motallebzadeh, A.A. Tameh, S.A.T. Zavareh, B. Farhood, A. Aliasgharzedeh, M. Mohseni, Neuroprotective effect of melatonin on radiation-induced oxidative stress and apoptosis in the brainstem of rats, J Cell Physiol, 235 (2020) 8791-8798.

[18] S. Rastogi, C. Haldar, Comparative effect of melatonin and quercetin in counteracting LPS induced oxidative stress in bone marrow mononuclear cells and spleen of Funambulus pennanti, Food Chem Toxicol, 120 (2018) 243-252.

[19] D.S. Salmanoglu, T. Gurpinar, K. Vural, N. Ekerbicer, E. Darıverenli, A. Var, Melatonin and L-carnitin improves endothelial disfunction and oxidative stress in Type 2 diabetic rats, Redox Biol, 8 (2016) 199204.

[20] L.d.S. Borges, A. Dermargos, E.P. da Silva Junior, E. Weimann, R.H. Lambertucci, E. Hatanaka, Melatonin decreases muscular oxidative stress and inflammation induced by strenuous exercise and stimulates growth factor synthesis, J Pineal Res, 58 (2015) 166-172.

[21] H. Ma, X. Wang, W. Zhang, H. Li, W. Zhao, J. Sun, M. Yang, Melatonin Suppresses Ferroptosis Induced by High Glucose via Activation of the Nrf2/HO-1 Signaling Pathway in Type 2 Diabetic Osteoporosis, Oxid Med Cell Longev, 2020 (2020) 9067610.

[22] A.M. Greenbaum, L.D. Revollo, J.R. Woloszynek, R. Civitelli, D.C. Link, N-cadherin in osteolineage cells is not required for maintenance of hematopoietic stem cells, Blood, 120 (2012) 295-302.

[23] B.S. Moriarity, G.M. Otto, E.P. Rahrmann, S.K. Rathe, N.K. Wolf, M.T. Weg, L.A. Manlove, R.S. LaRue, N.A. Temiz, S.D. Molyneux, K. Choi, K.J. Holly, A.L. Sarver, M.C. Scott, C.L. Forster, J.F. Modiano, C. Khanna, S.M. Hewitt, R. Khokha, Y. Yang, R. Gorlick, M.A. Dyer, D.A. Largaespada, A Sleeping Beauty forward genetic screen identifies new genes and pathways driving osteosarcoma development and metastasis, Nat Genet, 47 (2015) 615-624.

[24] X. Liu, M. Chen, J. Luo, H. Zhao, X. Zhou, Q. Gu, H. Yang, X. Zhu, W. Cui, Q. Shi, Immunopolarizationregulated 3D printed-electrospun fibrous scaffolds for bone regeneration, Biomaterials, 276 (2021) 121037.

[25] W. Fu, W. Hu, Y.-S. Yi, A. Hettinghouse, G. Sun, Y. Bi, W. He, L. Zhang, G. Gao, J. Liu, K. Toyo-Oka, G. Xiao, D.B. Solit, P. Loke, C.-J. Liu, TNFR2/14-3-3ع signaling complex instructs macrophage plasticity in 
inflammation and autoimmunity, J Clin Invest, (2021).

[26] C. Dou, J. Li, J. He, F. Luo, T. Yu, Q. Dai, Y. Chen, J. Xu, X. Yang, S. Dong, Bone-targeted pH-responsive cerium nanoparticles for anabolic therapy in osteoporosis, Bioact Mater, 6 (2021) 4697-4706.

[27] T. Vellai, How the amino acid leucine activates the key cell-growth regulator mTOR, Nature, (2021).

[28] A. Cipponi, D.L. Goode, J. Bedo, M.J. McCabe, M. Pajic, D.R. Croucher, A.G. Rajal, S.R. Junankar, D.N. Saunders, P. Lobachevsky, A.T. Papenfuss, D. Nessem, M. Nobis, S.C. Warren, P. Timpson, M. Cowley, A.C. Vargas, M.R. Qiu, D.G. Generali, S. Keerthikumar, U. Nguyen, N.M. Corcoran, G.V. Long, J.-Y. Blay, D.M. Thomas, MTOR signaling orchestrates stress-induced mutagenesis, facilitating adaptive evolution in cancer, Science, 368 (2020) 1127-1131.

[29] L. Xian, X. Wu, L. Pang, M. Lou, C.J. Rosen, T. Qiu, J. Crane, F. Frassica, L. Zhang, J.P. Rodriguez, J. Xiaofeng, Y. Shoshana, X. Shouhong, E. Argiris, W. Mei, C. Xu, Matrix IGF-1 maintains bone mass by activation of mTOR in mesenchymal stem cells, Nat Med, 18 (2012) 1095-1101.

[30] J. Yan, B. Ni, G. Sheng, Y. Zhang, Y. Xiao, Y. Ma, H. Li, H. Wu, C. Tu, Rhoifolin Ameliorates Osteoarthritis Regulating Autophagy, Front Pharmacol, 12 (2021) 661072.

[31] B. Stauch, L.C. Johansson, J.D. McCorvy, N. Patel, G.W. Han, X.-P. Huang, C. Gati, A. Batyuk, S.T. Slocum, A. Ishchenko, W. Brehm, T.A. White, N. Michaelian, C. Madsen, L. Zhu, T.D. Grant, J.M. Grandner, A. Shiriaeva, R.H.J. Olsen, A.R. Tribo, S. Yous, R.C. Stevens, U. Weierstall, V. Katritch, B.L. Roth, W. Liu, V. Cherezov, Structural basis of ligand recognition at the human MT melatonin receptor, Nature, 569 (2019) 284-288.

[32] T. Rui, H. Wang, Q. Li, Y. Cheng, Y. Gao, X. Fang, X. Ma, G. Chen, C. Gao, Z. Gu, S. Song, J. Zhang, C. Wang, Z. Wang, T. Wang, M. Zhang, J. Min, X. Chen, L. Tao, F. Wang, C. Luo, Deletion of ferritin H in neurons counteracts the protective effect of melatonin against traumatic brain injury-induced ferroptosis, J Pineal Res, 70 (2021) e12704.

[33] Z. Gou, X. Su, X. Hu, Y. Zhou, L. Huang, Y. Fan, J. Li, L. Lu, Melatonin improves hypoxic-ischemic brain damage through the Akt/Nrf2/Gpx4 signaling pathway, Brain Res Bull, 163 (2020) 40-48.

[34] G. Adami, K.G. Saag, Glucocorticoid-induced osteoporosis: 2019 concise clinical review, Osteoporos Int, 30 (2019) 1145-1156.

[35] G. Shen, H. Ren, Q. Shang, T. Qiu, X. Yu, Z. Zhang, J. Huang, W. Zhao, Y. Zhang, D. Liang, X. Jiang, Autophagy as a target for glucocorticoid-induced osteoporosis therapy, Cell Mol Life Sci, 75 (2018) 26832693.

[36] R. Rizzoli, E. Biver, Glucocorticoid-induced osteoporosis: who to treat with what agent?, Nat Rev Rheumatol, 11 (2015). 
[37] Q. Xing, J. Feng, X. Zhang, Semaphorin3B Promotes Proliferation and Osteogenic Differentiation of Bone Marrow Mesenchymal Stem Cells in a High-Glucose Microenvironment, Stem Cells Int, 2021 (2021) 6637176.

[38] G.-Y. Shen, H. Ren, Q. Shang, W.-H. Zhao, Z.-D. Zhang, X. Yu, J.-J. Huang, J.-J. Tang, Z.-D. Yang, D. Liang, X.-B. Jiang, Let-7f-5p regulates TGFBR1 in glucocorticoid-inhibited osteoblast differentiation and ameliorates glucocorticoid-induced bone loss, Int J Biol Sci, 15 (2019) 2182-2197.

[39] J. Li, N. Zhang, X. Huang, J. Xu, J.C. Fernandes, K. Dai, X. Zhang, Dexamethasone shifts bone marrow stromal cells from osteoblasts to adipocytes by C/EBPalpha promoter methylation, Cell Death Dis, 4 (2013) e832.

[40] N. Yang, H. Sun, Y. Xue, W. Zhang, H. Wang, H. Tao, X. Liang, M. Li, Y. Xu, L. Chen, L. Zhang, L. Huang, D. Geng, Inhibition of MAGL activates the Keap1/Nrf2 pathway to attenuate glucocorticoid-induced osteonecrosis of the femoral head, Clin Transl Med, 11 (2021) e447.

[41] Y. Mo, W. Lai, Y. Zhong, Z. Hu, M. You, M. Du, P. Wang, X. Wu, C. Chen, H. He, Z. Gao, Y. Xu, D. Wang, L. Cui, Y. Yang, TXNIP contributes to bone loss via promoting the mitochondrial oxidative phosphorylation during glucocorticoid-induced osteoporosis, Life Sci, 266 (2021) 118938.

[42] S.K. NaveenKumar, M. Hemshekhar, K. Kemparaju, K.S. Girish, Hemin-induced platelet activation and ferroptosis is mediated through ROS-driven proteasomal activity and inflammasome activation: Protection by Melatonin, Biochim Biophys Acta Mol Basis Dis, 1865 (2019) 2303-2316.

[43] P.M. LoRusso, Inhibition of the PI3K/AKT/mTOR Pathway in Solid Tumors, J Clin Oncol, 34 (2016) 3803-3815.

[44] D.A. Fruman, C. Rommel, PI3K and cancer: lessons, challenges and opportunities, Nat Rev Drug Discov, 13 (2014) 140-156.

[45] J. Yi, J. Zhu, J. Wu, C.B. Thompson, X. Jiang, Oncogenic activation of PI3K-AKT-mTOR signaling suppresses ferroptosis via SREBP-mediated lipogenesis, Proc Natl Acad Sci U S A, 117 (2020) 3118931197.

[46] R. Zhao, L. Tao, S. Qiu, L. Shen, Y. Tian, Z. Gong, Z.B. Tao, Y. Zhu, Melatonin rescues glucocorticoidinduced inhibition of osteoblast differentiation in MC3T3-E1 cells via the PI3K/AKT and BMP/Smad signalling pathways, Life Sci, 257 (2020) 118044.

[47] M. Chen, E. Cecon, A. Karamitri, W. Gao, R. Gerbier, R. Ahmad, R. Jockers, Melatonin MT and MT receptor ERK signaling is differentially dependent on G and G proteins, J Pineal Res, 68 (2020) e12641.

[48] B. Tang, J. Zhu, S. Fang, Y. Wang, R. Vinothkumar, M. Li, Q. Weng, L. Zheng, Y. Yang, R. Qiu, M. Xu, Z. Zhao, J. Ji, Pharmacological inhibition of MELK restricts ferroptosis and the inflammatory response in colitis and colitis-propelled carcinogenesis, Free Radic Biol Med, 172 (2021) 312-329. 

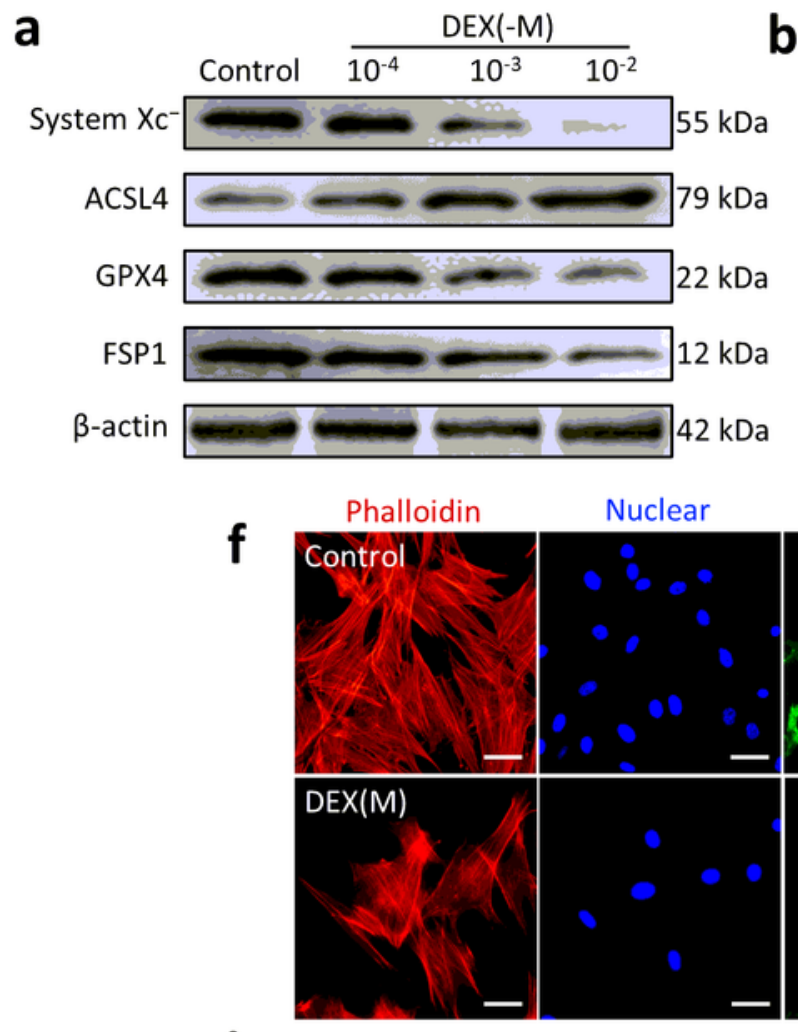

h
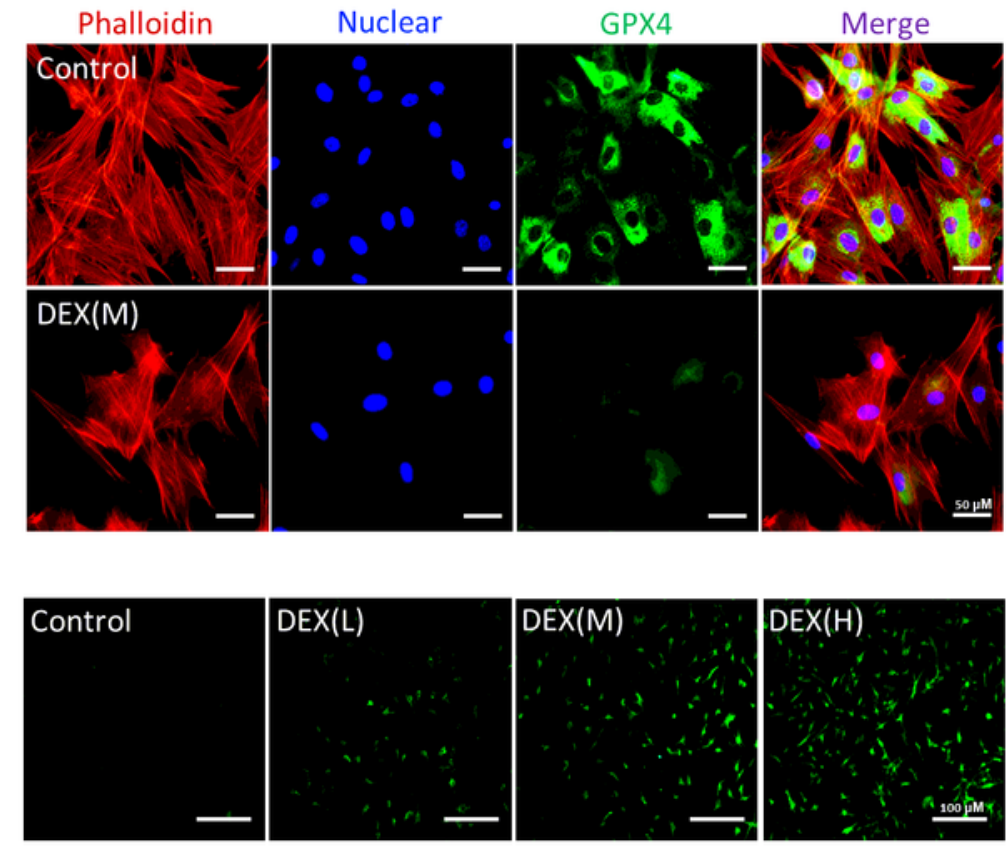

d e

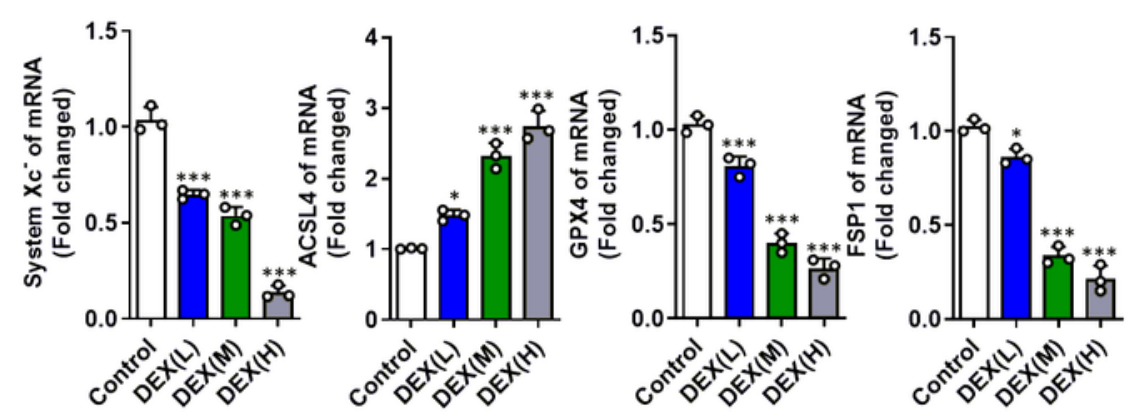

g
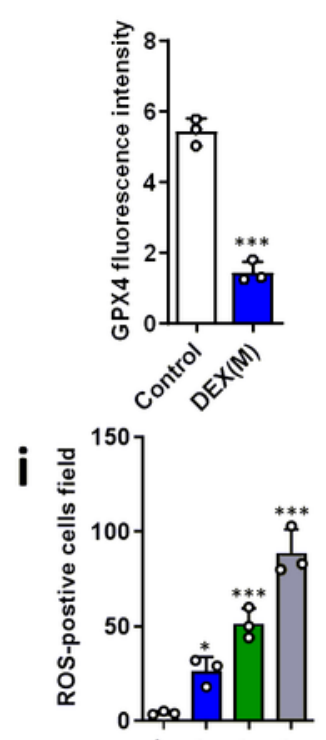

(1)

000

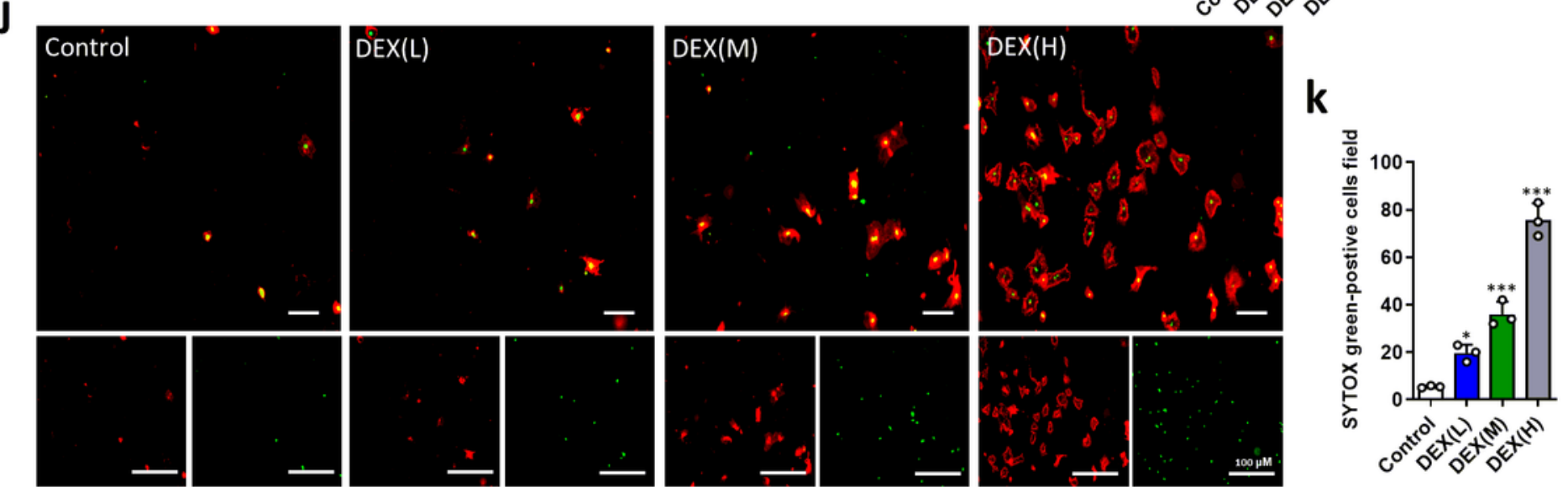

Figure 1

DEX activates the ferroptotic pathway of BMSCs. (a-e) BMSCs were treated with various concentrations of DEX for $24 \mathrm{~h}$ and the expressions of system Xc-, ACSL4, GPX4, and FSP1were analyzed by western blot and qRT-PCR. (f) Images of immunofluorescence staining of GPX4 in BMSCs after treatment with 
DEX(10-3M) for $72 \mathrm{~h}$. (g) Quantification of the fluorescence intensity of GPX4 immunofluorescence positively stained cells. (h) ROS staining was performed to test the correlation between different concentrations of DEX and the level of oxidative stress. (i) Quantitative analysis of the number of ROSpositive cells per field in (h). (j) Annexin V-mCherry/SYTOX Green detection kit was used to detect cell death. (k) Quantitative analysis of the percentage of SYTOX green-positive cells in (j). These studies were performed at least 3 biological replicates. Data represent mean \pm S.D. $(n=3) .{ }^{*} P<0.05,{ }^{\star *} P<0.01$, $\star * * \mathrm{P}<0.005$ compared with control group.

a

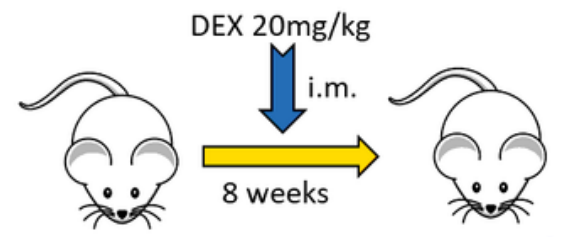

SIOP-Model
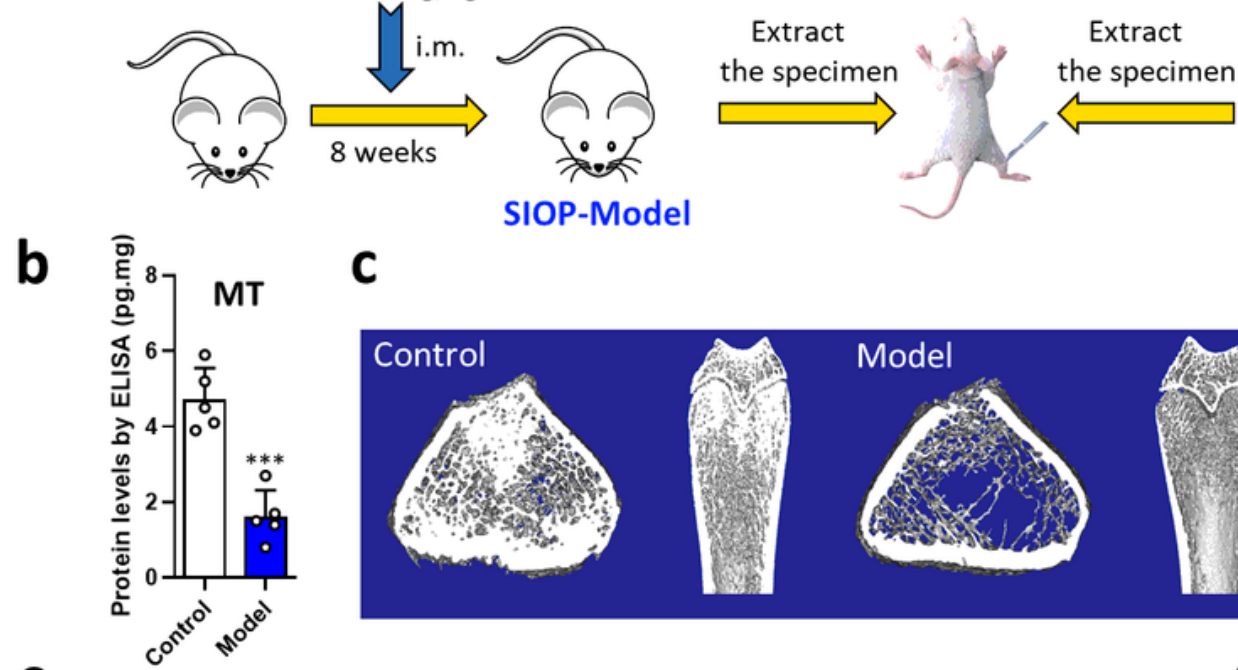

C
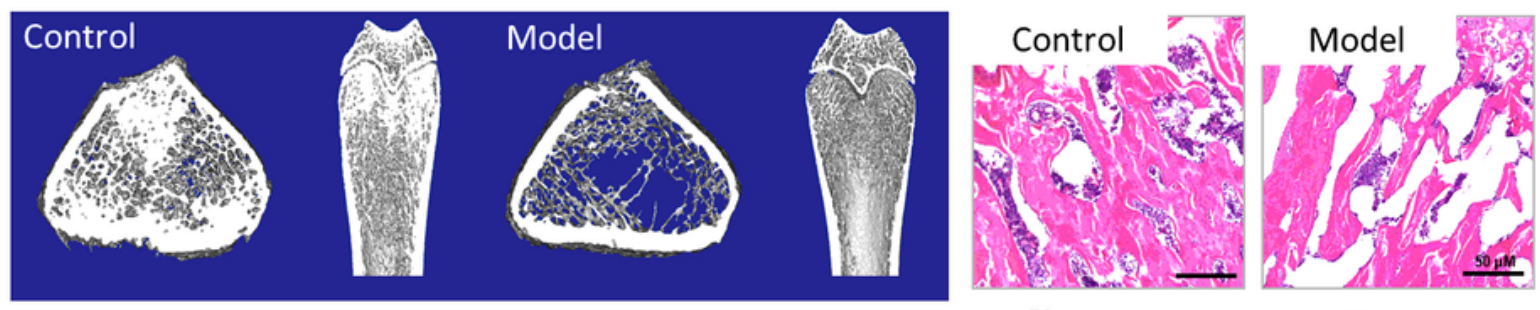

e

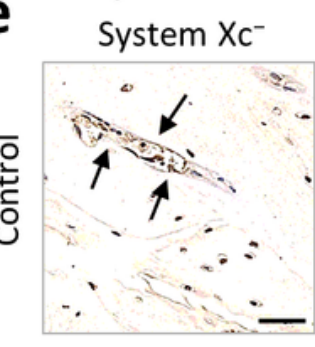

GPX4
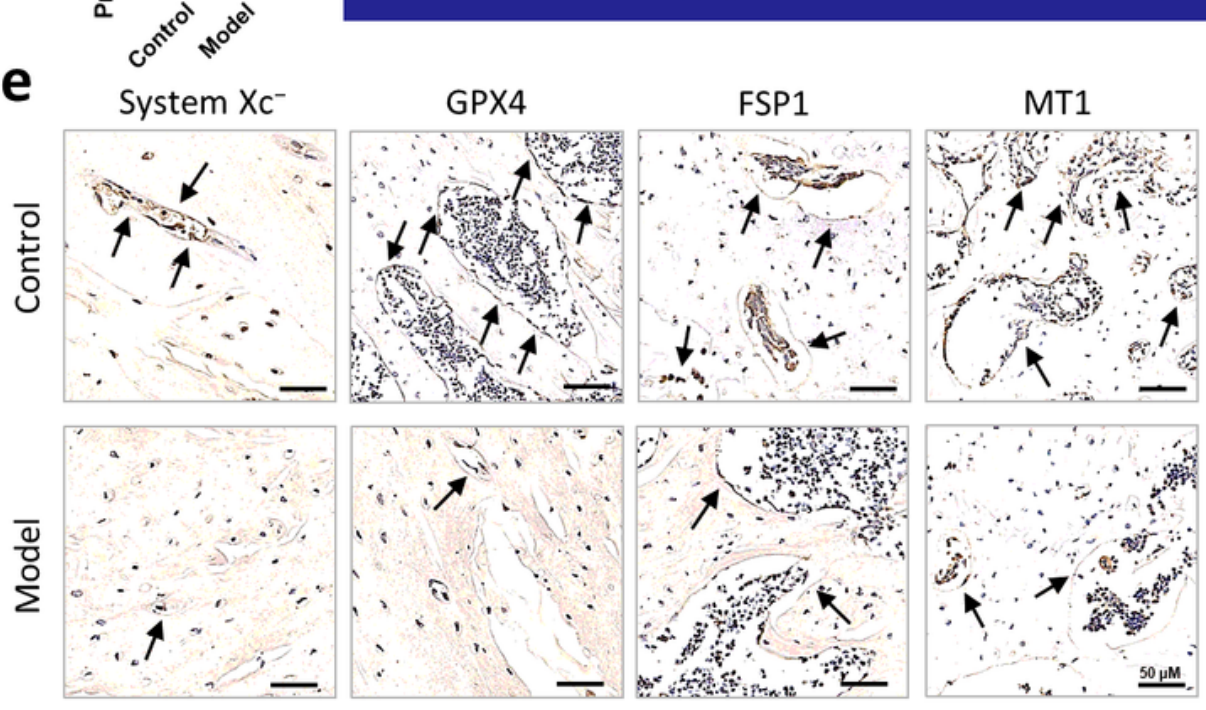

FSP1
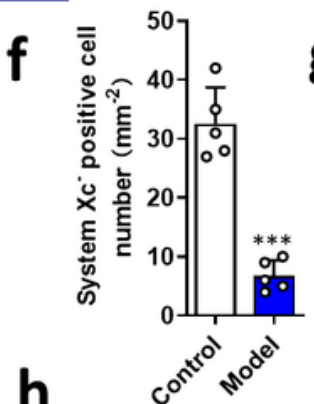

h
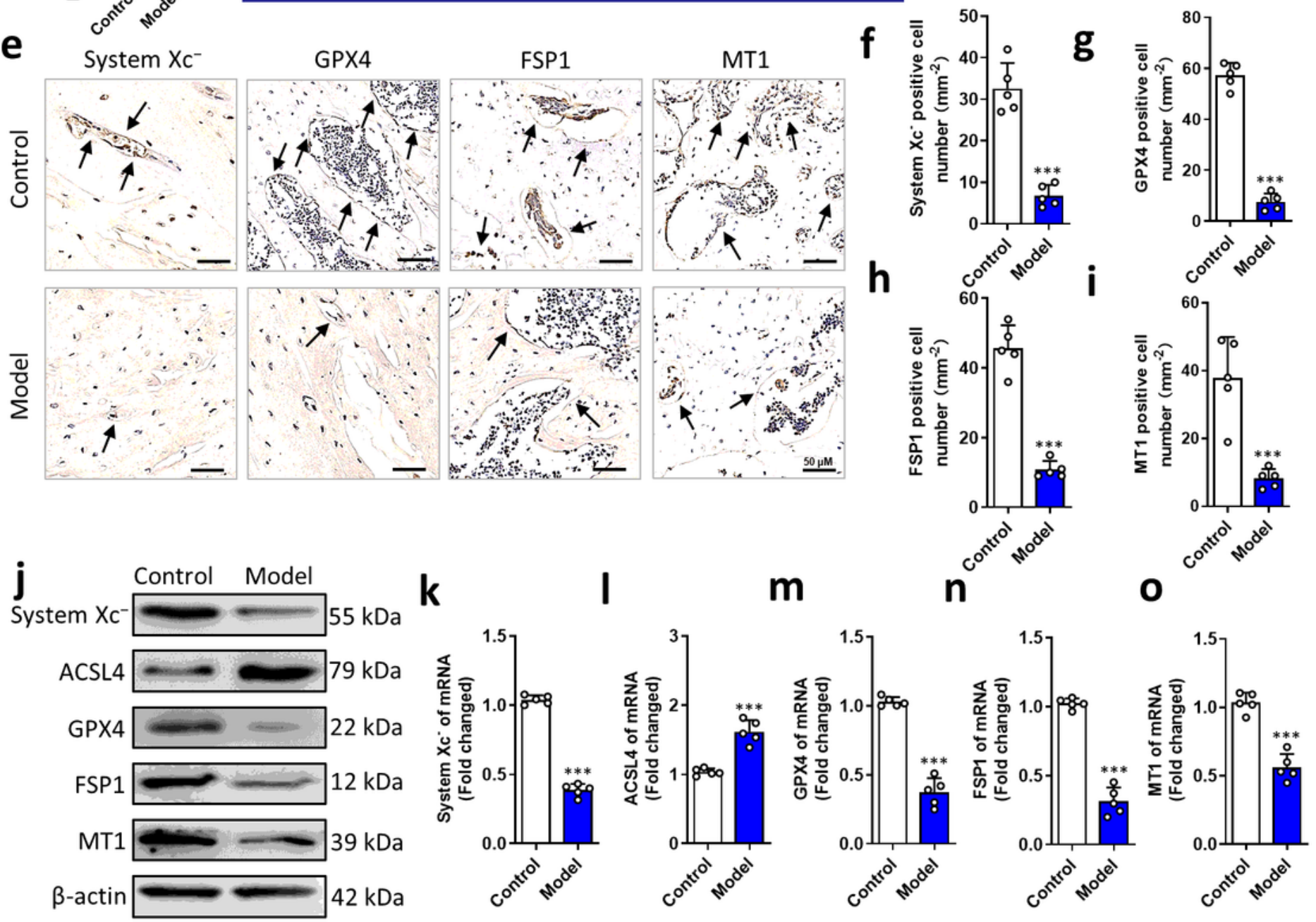

0
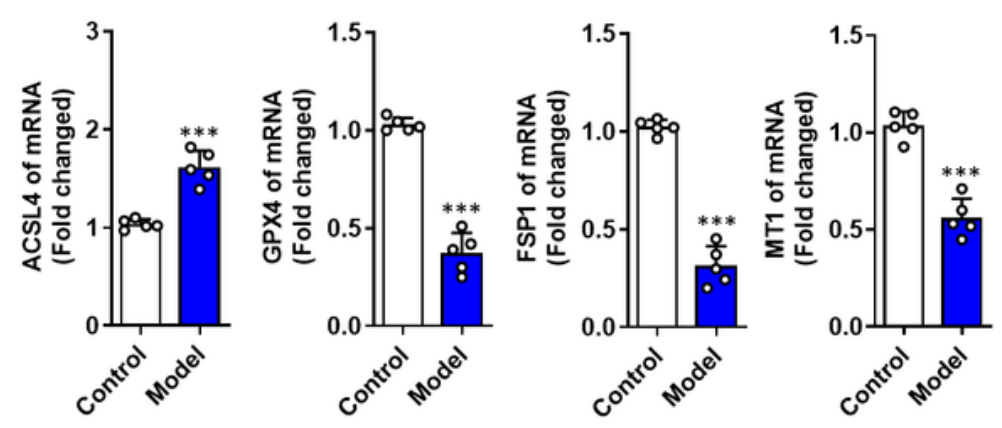
Figure 2

DEX activates the ferroptotic pathway in SIOP. (a) The timeline of the GC-induced SIOP model. (b) Quantification of the protein level of MT by ELASA. (c) Images of Micro-CT. (d) H\&E staining. (e) IHC staining of system xc-, GPX4, FSP1 and MT1, the IHC-positive cells were marked with black arrows. (f-i) Quantitative analysis of the number of the IHC-positive cells in (e). (j-o) The expressions of system xc-, ACSL4, GPX4, FSP1 and MT1 were analyzed by western blot and qRT-PCR. These studies were performed at least 3 biological replicates. Data represent mean \pm S.D. $(n=5) .{ }^{*} P<0.05,{ }^{\star \star} P<0.01,{ }^{\star \star *} P<0.005$ compared with control group.

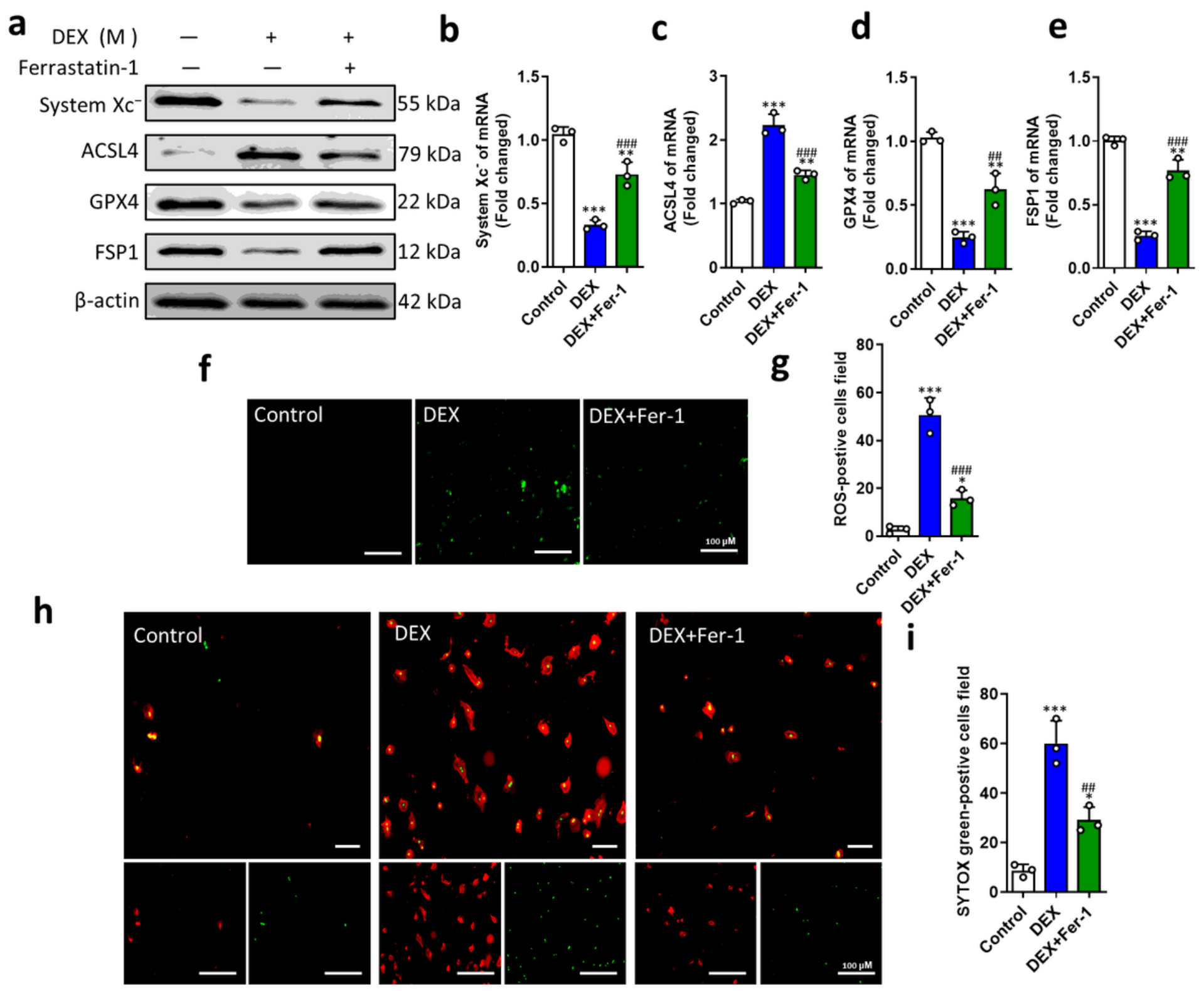

\section{Figure 3}

Ferroptosis inhibitor regulates the expression of related proteins in BMSCs. (a-e) Western blot and qRTPCR results for the expressions of system xC-, ACSL4, GPX4, and FSP1 were pretreated with ferroptosis inhibitor Ferrastatin-1 for 24 h; DEX(10-3M) was then added for 24 h. (f) ROS staining was performed to 
test the level of oxidative stress in control group, DEX(10-3M) group and DEX+Fer-1 group. (g) Quantitative analysis of the number of ROS-positive cells per field in (f). (h) Annexin V-mCherry/SYTOX Green detection kit was used to detect cell death. (i) Quantitative analysis of the percentage of SYTOX green-positive cells in (h). These studies were performed at least 3 biological replicates. Data represent

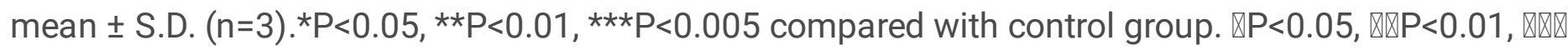
$\mathrm{P}<0.005$ compared with DEX group.

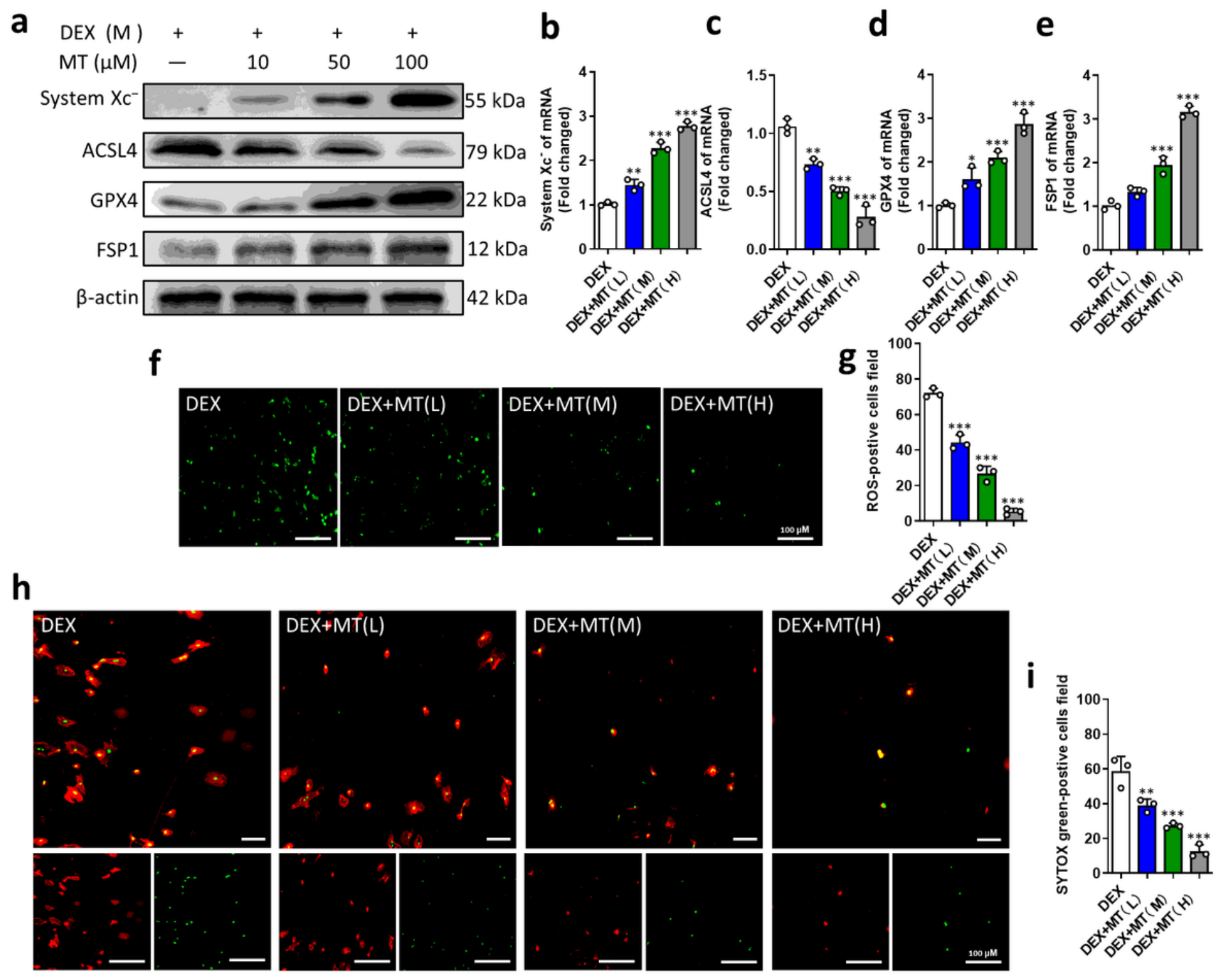

\section{Figure 4}

MT can reduce the ferroptosis level in BMSCs induced by DEX. (a-e) Western blot and qRT-PCR results for the expressions of system xc-, ACSL4, GPX4, and FSP1 were pretreated with various concentrations of MT for 24 h; DEX(10-3M) was then added for $24 \mathrm{~h}$. (f) ROS staining was performed to test the correlation between different concentrations of MT and the level of oxidative stress. (g) Quantitative analysis of the number of ROS-positive cells per field in (f). (h) Annexin V-mCherry/SYTOX Green detection kit was used to detect cell death. (i) Quantitative analysis of the percentage of SYTOX green-positive cells in (h). These 
studies were performed at least 3 biological replicates. Data represent mean \pm S.D. $(n=3)$. ${ }^{*}<0.05$, ** $\mathrm{P}<0.01,{ }^{* * *} \mathrm{P}<0.005$ compared with DEX group.
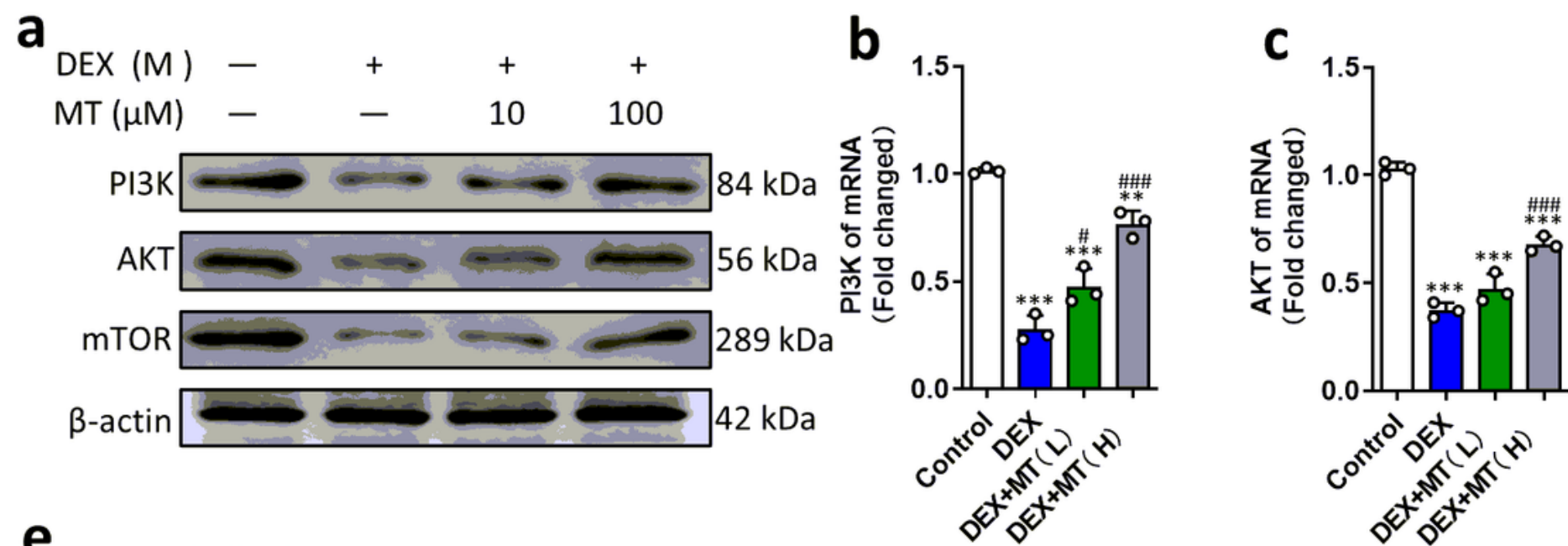

$\mathbf{e}$
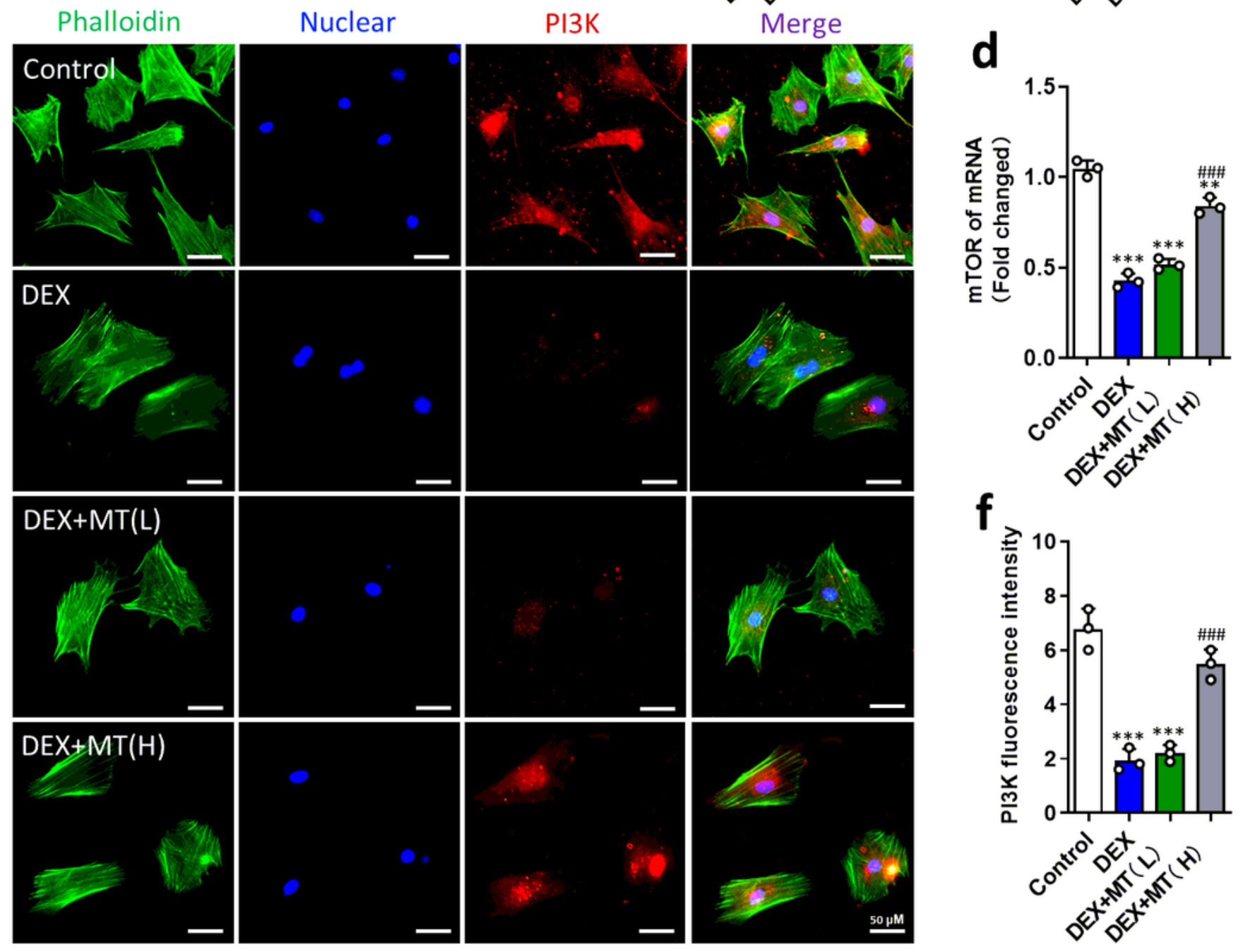

Figure 5

MT reduces ferroptosis in DEX-induced BMSCs by activating the PI3K-AKT-mTOR pathway. (a-d) Western blot and qRT-PCR results for the expressions of PI3K, AKT, and mTOR were pretreated with various concentrations of MT for 24 h; DEX(10-3M) was then added for 24 h. (e) Images of immunofluorescence 
staining of PI3K in BMSCs. (f) Quantification of the fluorescence intensity of PI3K immunofluorescence positively stained cells. These studies were performed at least 3 biological replicates. Data represent

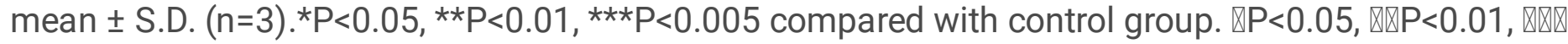
$\mathrm{P}<0.005$ compared with DEX group.
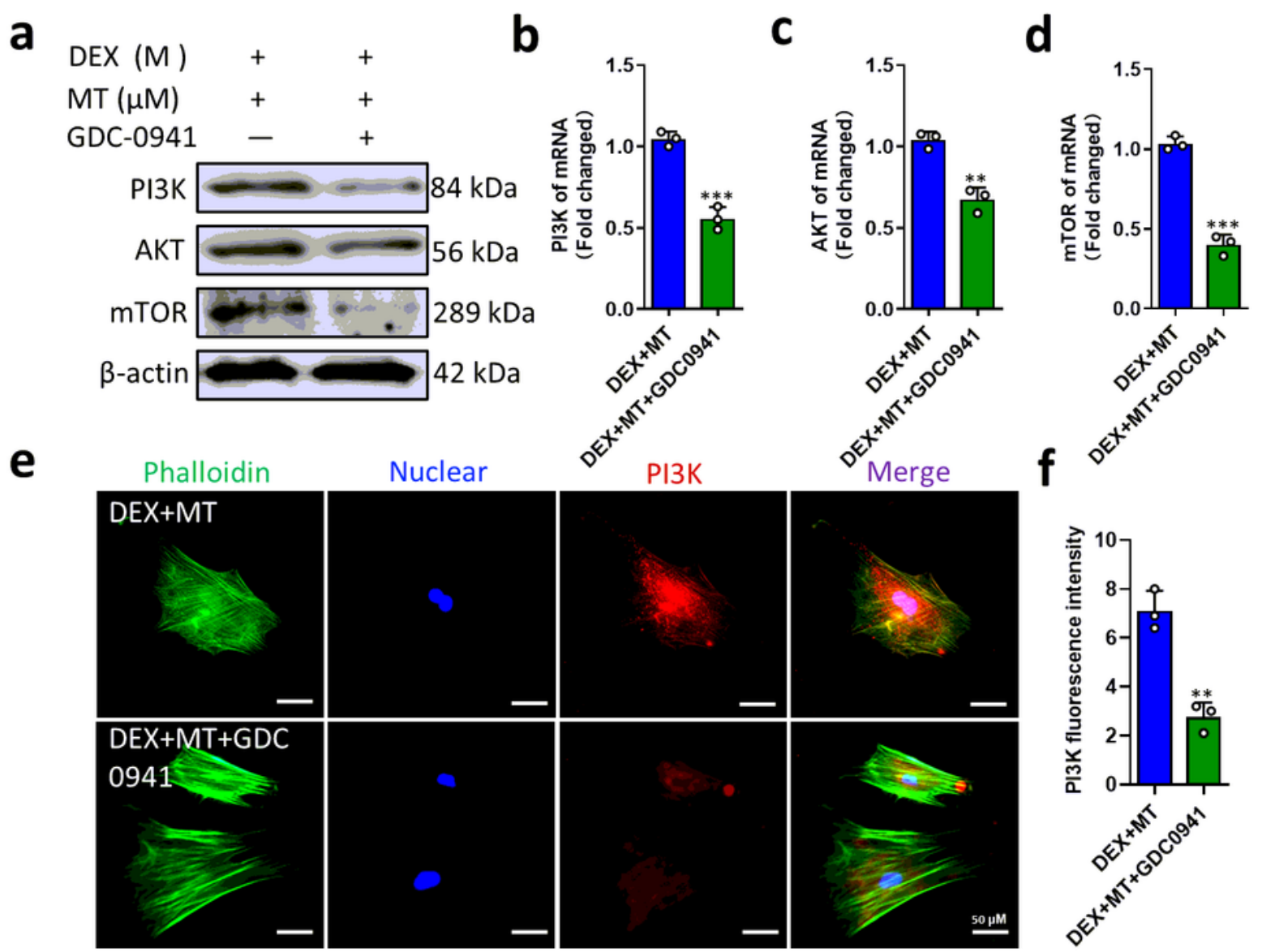

\section{g}
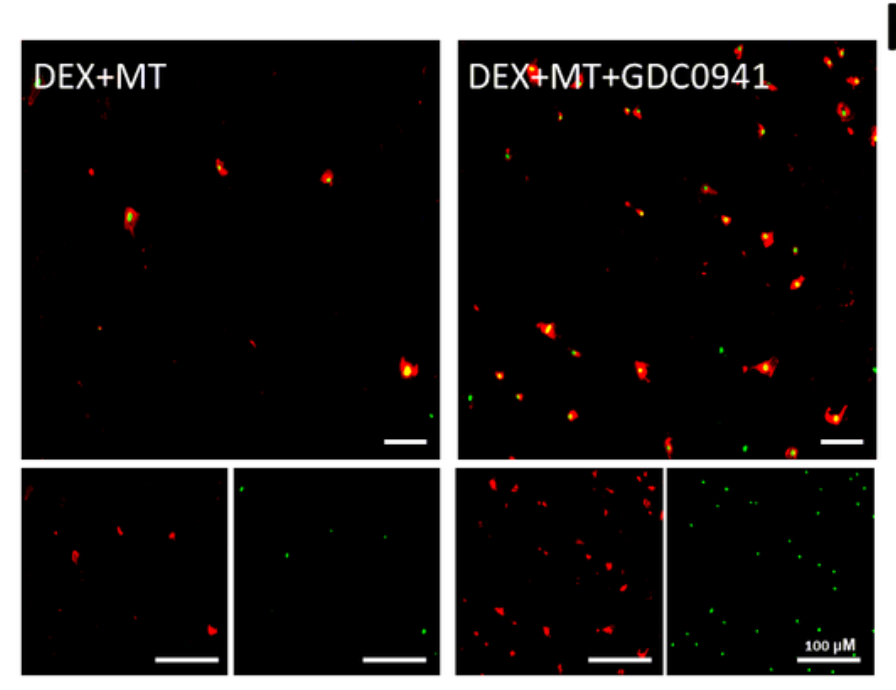

h
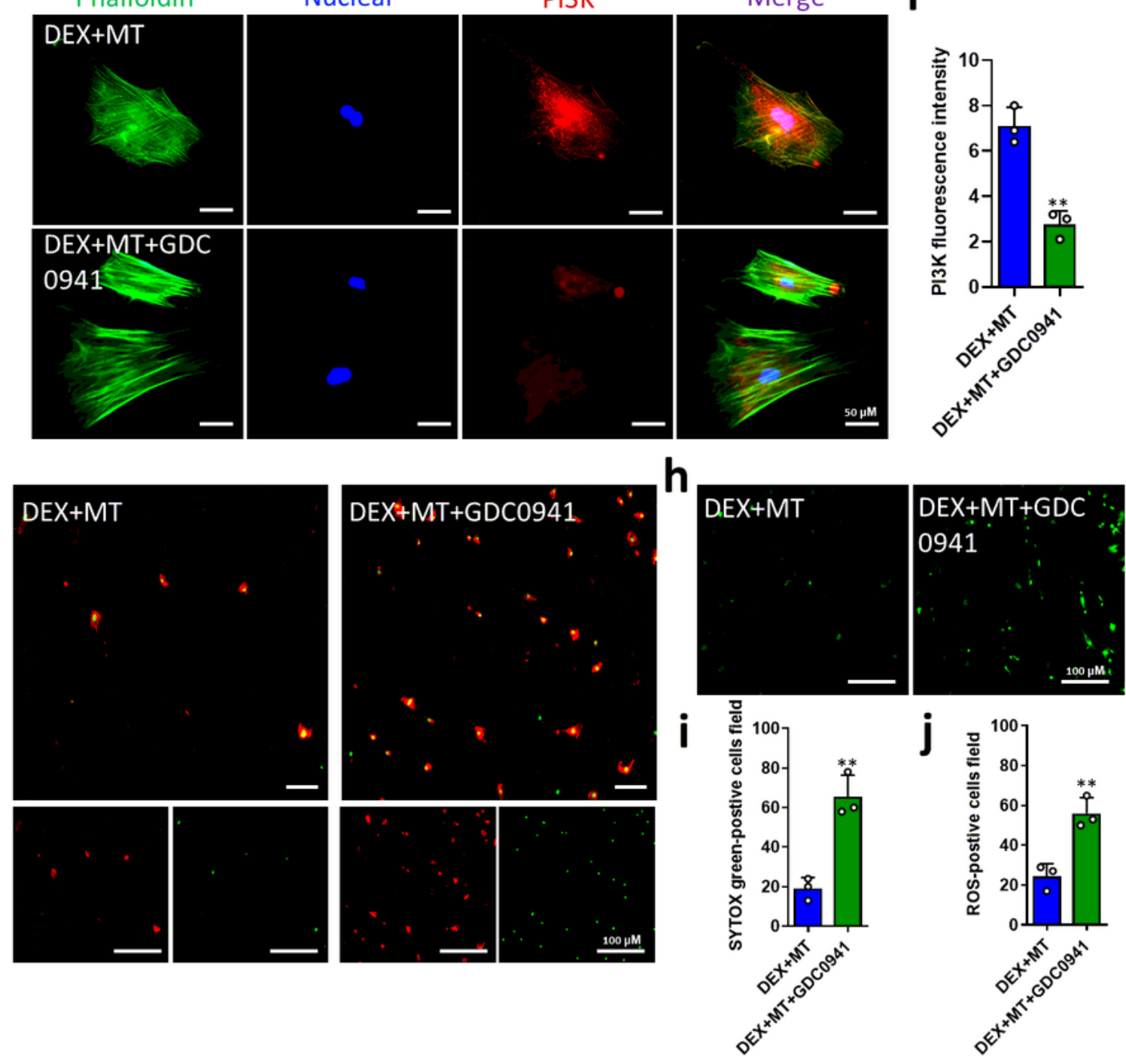

Figure 6 
The positive regulatory effect of MT was significantly weakened with the use of PI3K inhibitors. (a-d) Western blot and qRT-PCR results for the expressions of PI3K, AKT, and mTOR were pretreated with GDC0941 and MT for 24 h; DEX(10-3M) was then added for 24 h. (e) Images of immunofluorescence staining of PI3K in BMSCs. (f) Quantification of the fluorescence intensity of PI3K immunofluorescence positively stained cells. (g) Annexin V-mCherry/SYTOX Green detection kit was used to detect cell death. (i) Quantitative analysis of the percentage of SYTOX green-positive cells in (g). (h) ROS staining was performed to test the level of oxidative stress in DEX+MT group and DEX+MT+GDC0941 group. (j) Quantitative analysis of the number of ROS-positive cells per field in (h). These studies were performed at least 3 biological replicates. Data represent mean \pm S.D. $(n=3) .{ }^{*} P<0.05, \star \star P<0.01,{ }^{\star} * \star * P<0.005$ compared with DEX+MT group. 
a
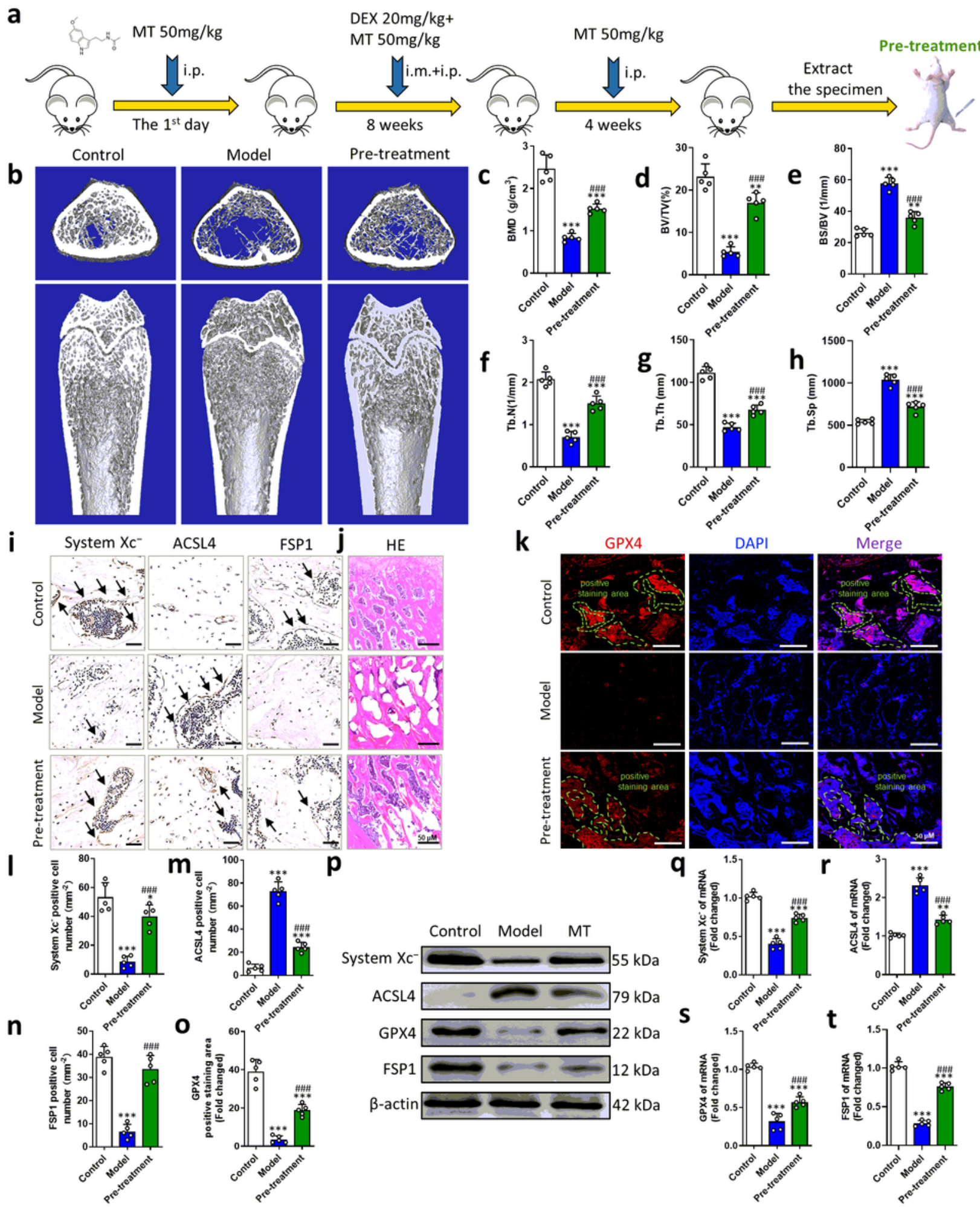

Figure 7

Pre-treatment with MT alleviates DEX-induced SIOP in vivo. (a) The timeline of the DEX-induced SIOP model and administration of MT in vivo. (b) Images of Micro-CT. (c) BMD (g/cm3). (d) BV/TV (\%). (e) BS/BV(1/mm). (f) Tb.N(1/mm). (g) Tb.Th (mm). (h) Tb.Sp (mm). (i) IHC staining of system xc-, ACSL4 and FSP1, the IHC-positive cells were marked with black arrows. (I-n) Quantitative analysis of the number of the IHC-positive cells in (i). (j) H\&E staining. (k) Images of immunofluorescence staining of GPX4 in 
bone tissues. (o) Quantitative analysis of the area of GPX4-positive stains. (p-t) The expressions of system XC-, ACSL4, GPX4 and FSP1 were analyzed by western blot and qRT-PCR in bone tissues between model and Pre-treatment groups. These studies were performed at least 3 biological replicates. Data represent mean \pm S.D. $(n=5) .{ }^{*} P<0.05,{ }^{*} P<0.01,{ }^{* \star *} P<0.005$ compared with control group. $\otimes P<0.05, \mathbb{} \otimes$ $\mathrm{P}<0.01$, $\mathbb{X \otimes} \otimes \mathrm{P}<0.005$ compared with model group.

a
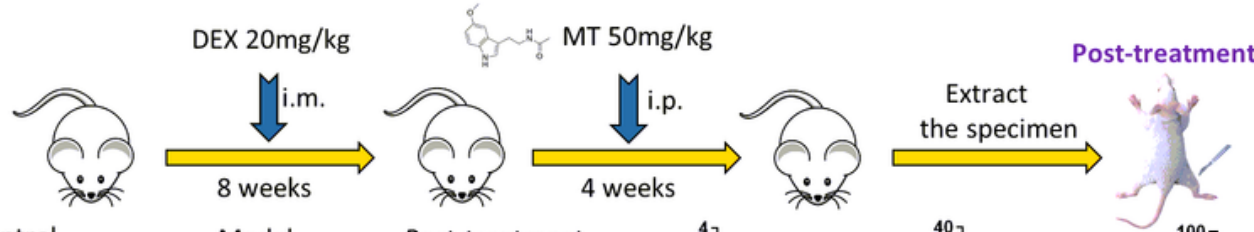

b
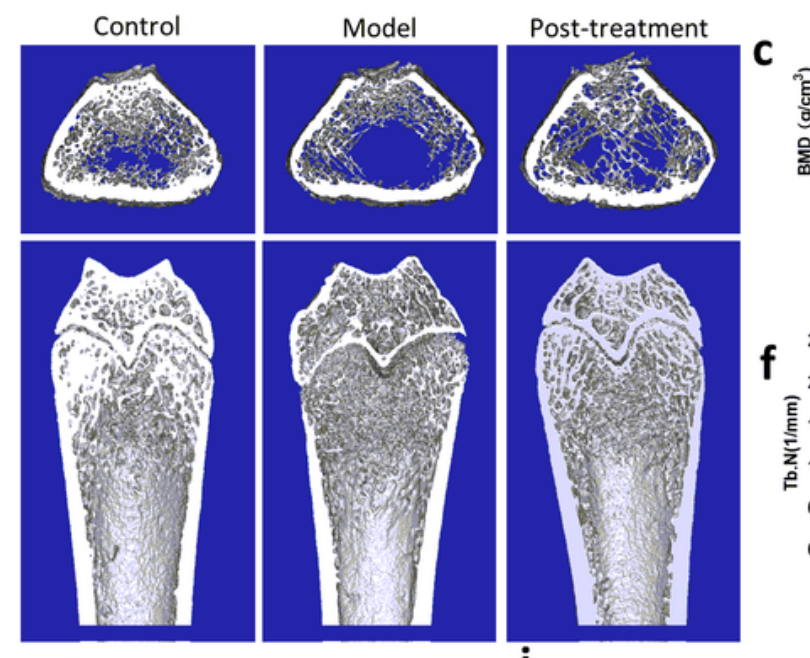

i

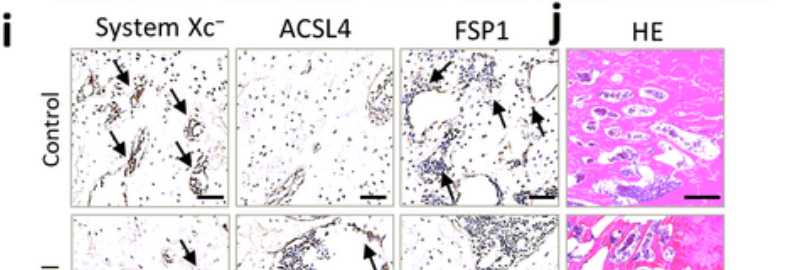

ACSL4

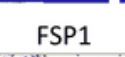

HE
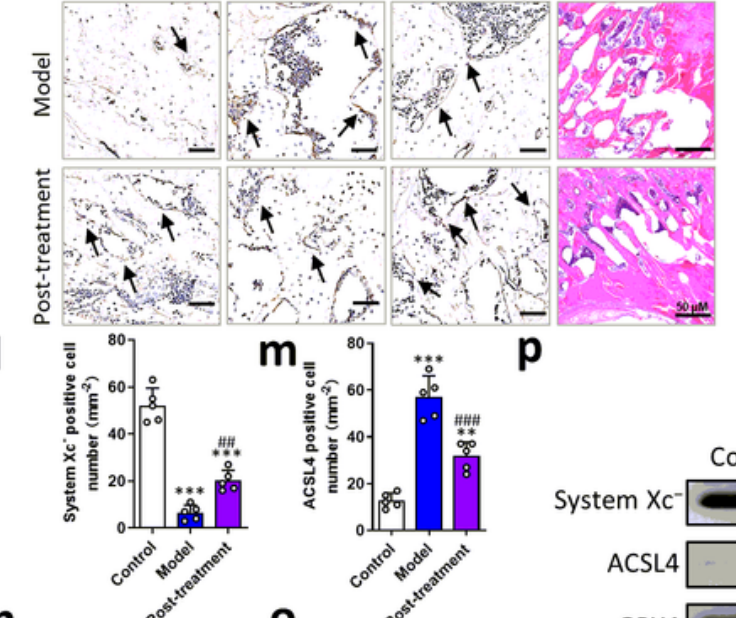

n

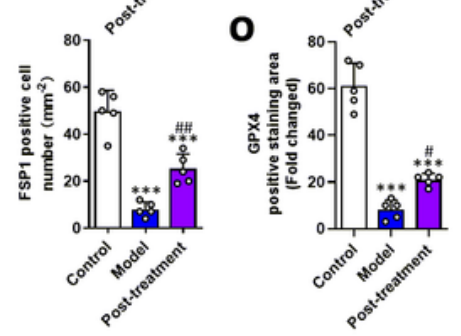

p
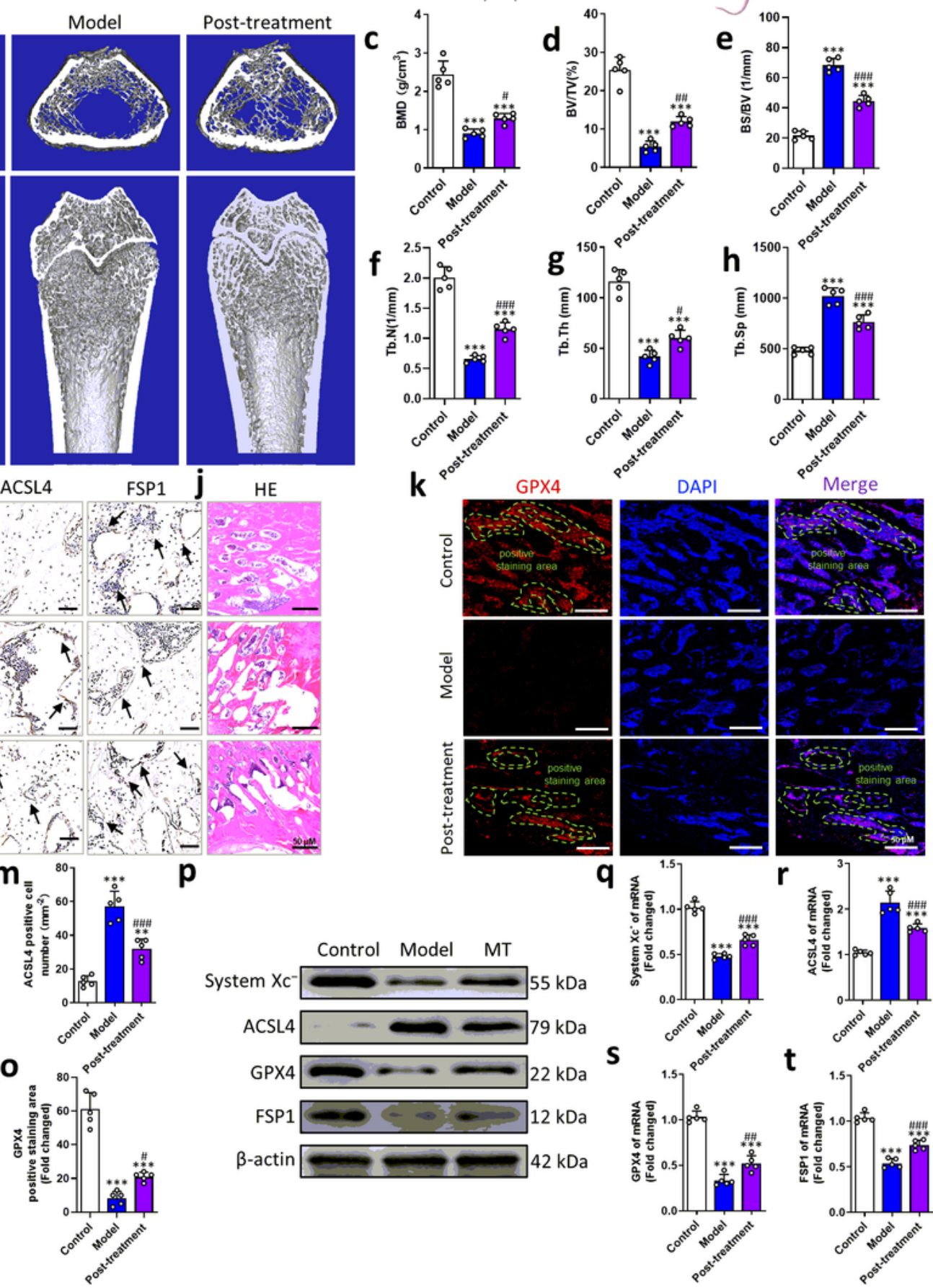

Figure 8 
Even after DEX-induced ferroptotic effects begin, MT can still improve SIOP. (a) The timeline of the DEXinduced SIOP model and administration of MT in vivo. (b) Images of Micro-CT. (c) BMD(g/cm3). (d) BV/TV (\%). (e) BS/BV(1/mm). (f) Tb.N(1/mm). (g) Tb.Th (mm). (h) Tb.Sp (mm). (i) IHC staining of system xc-, ACSL4 and FSP1, the IHC-positive cells were marked with black arrows. (I-n) Quantitative analysis of the number of the IHC-positive cells in (i). (j) H\&E staining. (k) Images of immunofluorescence staining of GPX4 in bone tissues. (o) Quantitative analysis of the area of GPX4-positive stains. (p-t) The expressions of system xc-, ACSL4, GPX4 and FSP1 were analyzed by western blot and qRT-PCR in bone tissues between model and Post-treatment groups. These studies were performed at least 3 biological replicates. Data represent mean \pm S.D. $(n=5) .{ }^{*} P<0.05,{ }^{\star} * P<0.01,{ }^{\star} * \star P<0.005$ compared with control group.

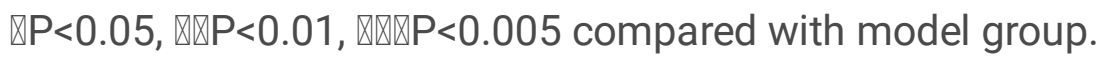

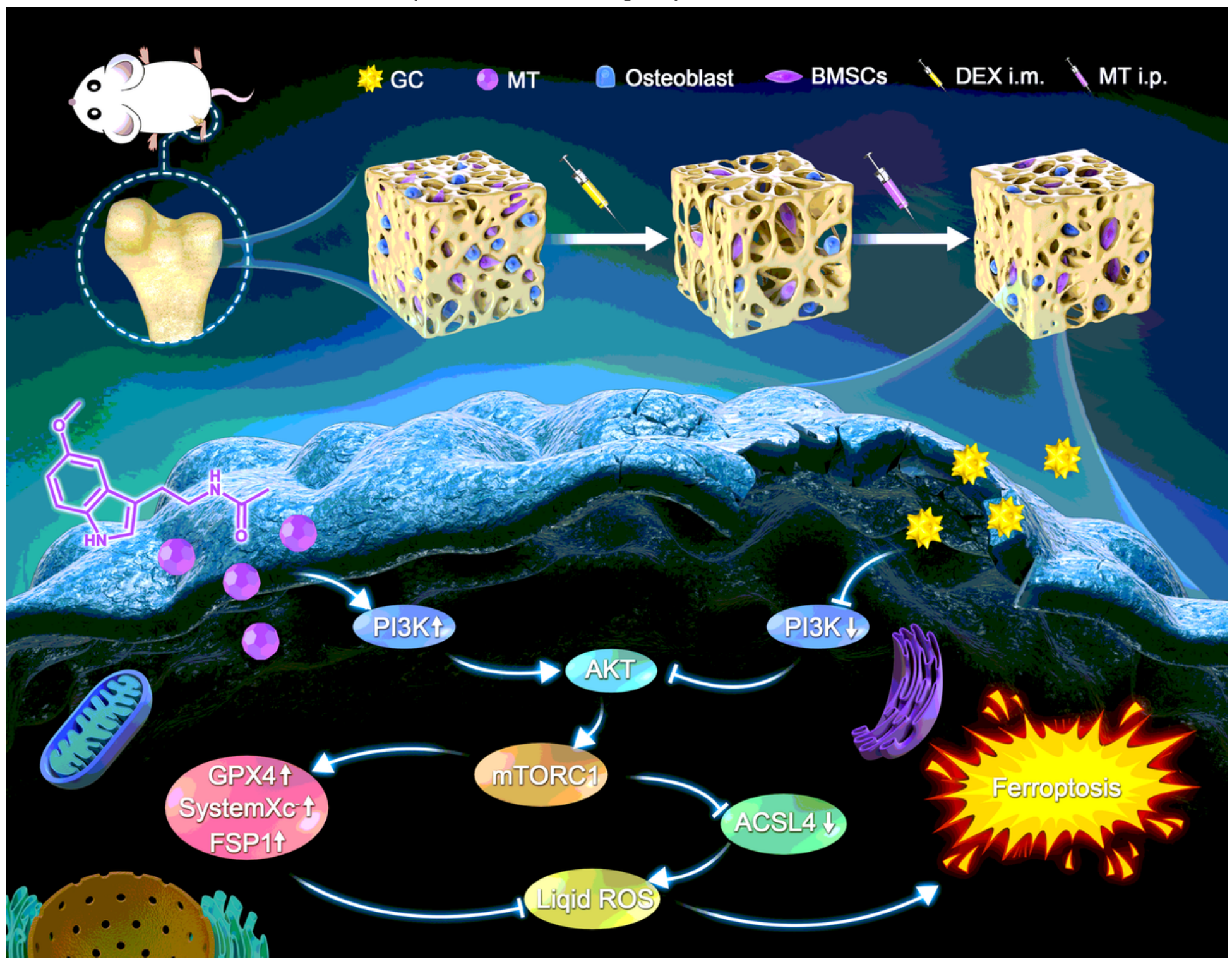

\section{Figure 9}

Schematic illustration of MT-mediated protection from GC-induced ferroptosis. Melatonin inhibits the ferroptotic pathway in rat bone marrow mesenchymal stem cells by activating the PI3K-AKT-mTOR signaling axis to attenuate steroid-induced osteoporosis. 


\section{Supplementary Files}

This is a list of supplementary files associated with this preprint. Click to download.

- Supplementarydata.docx 